Research Article

\title{
Adaptive Fixed-Time Tracking Consensus Control for Multiagent Nonlinear Pure-Feedback Systems with Performance Constraints
}

\author{
Pinwei Li $(\mathbb{D}$, Jiyang Dai $\mathbb{D}$, and Jin Ying $(\mathbb{D}$ \\ School of Information Engineering, Nanchang Hangkong University, Nanchang 330063, China \\ Correspondence should be addressed to Jiyang Dai; djiyang@163.com
}

Received 8 August 2020; Revised 9 April 2021; Accepted 26 April 2021; Published 18 May 2021

Academic Editor: Xianming Zhang

Copyright (c) 2021 Pinwei Li et al. This is an open access article distributed under the Creative Commons Attribution License, which permits unrestricted use, distribution, and reproduction in any medium, provided the original work is properly cited.

\begin{abstract}
This paper investigates adaptive fixed-time tracking consensus control problems for multiagent nonlinear pure-feedback systems with performance constraints. Compared with existing results of first/second/high-order multiple agent systems, the studied systems have more complex nonlinear dynamics with each agent being modeled as a high-order pure-feedback form. The mean value theorem is introduced to address the problem of nonaffine structure in nonlinear pure-feedback systems. Meanwhile, radial basis function neural networks (RBFNNs) are employed to approximate unknown functions. Furthermore, a constraint variable is used to guarantee that all local tracking errors are within the prescribed boundaries. It is shown that, by utilizing the proposed consensus control protocol, each tracking consensus error can converge into a neighborhood around zero within designed fixed time, the tracking consensus performance can be ensured during the whole process, and all signals in the investigated systems are bounded. Finally, two simulations are performed and the results demonstrate the effectiveness of the proposed control strategy.
\end{abstract}

\section{Introduction}

In the past decade, the finite-time consensus control has attracted considerable attention due to its unique advantages in faster convergence rate and higher precision [1] and its wide applications in unmanned aerial vehicles, autonomous underwater vehicles, surface vessels, and so forth [2-5].

Finite-time control was first proposed in 1965, and many related research results emerged in the subsequent decades. A variety of finite-time control protocols [6-11] have developed to address the different agents' dynamics in multiagent systems (MASs). For example, in [12, 13], finite-time consensus control for first- and second-order MASs was proposed, respectively, by using the principle of homogeneity [14]. In [15], a finite-time control for MASs with double integrator dynamics was proposed by utilizing power integrator technique. Furthermore, two classes of finite-time control protocols for MASs composed of first-order and second-order integrator agents were developed by combining the homogeneous domination method with adding a power integrator method. Thereafter, to solve the consensus control problems of high-order uncertain MASs, several finite-time control strategies were proposed in [16-18]. Even so, there is a limitation for the finite-time control results that the convergence time is strongly dependent on the initial states of the systems; in other words, once the initial states are far away from the equilibrium point, the convergence time will increase as a result. As a matter of fact, it is much more reasonable for a predicable convergence time.

In 2012, to address the limitation of finite-time control, Polyakov [19] proposed fixed-time stability control, which can allow the convergence time of systems have nothing to do with its initial states. Subsequently, many related research results [20-29] for MASs have been carried out. It can be found that most of the existing fixed-time consensus control results are focused on MASs with first-order or secondorder, and only a few focus on high-order MASs. Although, in [24-27], the fixed-time control strategies have considered high-order MASs; they are not general enough, since their results apply only to strict-feedback nonlinear systems. However, compared with lower-triangular systems and strict-feedback nonlinear systems, the pure-feedback nonlinear systems [30-32] are more general systems. On the other hand, in many practical industrial systems, there are 
some requirements for performance constraint. For example, unknown dead-zone inputs [33], output-constrained [34], preassigned transient performance [35], and other measures are taken to prevent system damage. In [36], the authors investigated the adaptive tracking control problem for a class of nonlinear time-delay systems in the presence of input and tracking error constraints.

Motivated by the above observations, in this paper, we focus on addressing the problem of adaptive fixed-time tracking consensus control for a class of pure-feedback uncertain nonlinear MASs with performance constraint. In the design, to solve the problem of nonaffine structure in nonlinear pure-feedback systems, we employ mean value theorem. Meanwhile, RBF neural networks are used to compensate for uncertain nonlinear terms and some functions that are difficult to calculate induced from the controller design procedure. Furthermore, a predefined boundary function is utilized to cope up with the requirement of the prescribed performance. Finally, based on Lyapunov stability theorem, fixed-time control theory, and graph theory, a novel fixed-time consensus control strategy is designed for the considered pure-feedback nonlinear MASs, which can guarantee that all the local tracking errors are within the range of the predefined boundary function and can converge to the neighborhood of the origin in fixed time and all signals in MASs are bounded.

The main contributions of this paper can be summarized as follows:

(1) To our best knowledge, this paper is the first attempt to solve the problem of adaptive fixed-time consensus control for MASs with nonlinear pure-feedback. Compared with some existing results [16-18, 24-27] on high-order MASs with nonlinear strict-feedback, the investigated pure-feedback nonlinear MASs are more general.

(2) By introducing RBF neural networks, the uncertain nonlinear iterms in each agent's dynamics can be approximated infinitely. Therefore, the designed controller in this paper is more versatile and robust.

(3) Based on backstepping algorithm and Lyapunov theorem, a novel fixed-time consensus control protocol design method is proposed. Meanwhile, the tracking performance is governed by the predefined boundary function.

The rest of this paper is arranged as follows. Section 2 gives problem description and preliminaries. Section 3 presents the detailed design process of fixed-time controller. In Section 4, the stability analysis will be given. In Section 5, the validity of the proposed control scheme is proven by two simulations. Finally, the conclusion of this work is summarized in Section 6.

\section{Problem Description and Preliminaries}

2.1. Problem Statement. The considered nonlinear MASs consist of $M$ followers, labeled as agents 1 to $M$, and a leader, labeled as an agent 0 under a directed communication graph topology. The dynamic models of $M$ followers can be described as the form of the following pure-feedback nonlinear systems:

$$
\left\{\begin{array}{l}
\dot{x}_{i, k}(t)=f_{i, k}\left(\bar{x}_{i, k}(t), x_{i, k+1}(t)\right)+d_{i, k}\left(\bar{x}_{i, k}, t\right), \\
\dot{x}_{i, n_{i}}(t)=f_{i, n_{i}}\left(\bar{x}_{i, n_{i}}(t), u_{i}(t)\right)+d_{i, n_{i}}\left(\bar{x}_{i, n_{i}}, t\right), \\
y_{i}(t)=x_{i, 1}(t)
\end{array}\right.
$$

where $\quad i=1, \ldots, M, \quad k=1, \ldots, n_{i}-1, \quad \bar{x}_{i, k}=\left[x_{i, 1}\right.$ $\left.(t), \ldots, x_{i, k}(t)\right]^{T} \in R^{k}$ with $k=1, \ldots, n_{i}, \quad u_{i}(t) \in R$, and $y_{i}(t) \in R$ are system state variables, system input, and system output, respectively; $f_{i, k}(\cdot)$ are unknown smooth nonaffine functions; $d_{i, k}(\cdot)$ are unknown but bounded disturbances.

We utilize the mean value theorem to solve the nonaffine structures in pure-feedback systems:

$$
\begin{aligned}
f_{i, k}\left(\bar{x}_{i, k}, x_{i, k+1}\right) & =f_{i, k}\left(\bar{x}_{i, k}, x_{i, k 0}\right)+g_{i, k} \cdot\left(x_{i, k+1}-x_{i, k 0}\right), \\
f_{i, n_{i}}\left(\bar{x}_{i, n_{i}}, u_{i}\right) & =f_{i, n_{i}}\left(\bar{x}_{i, n_{i}}, x_{i, n_{i} 0}\right)+g_{i, n_{i}} \cdot\left(u_{i}-x_{i, n_{i} 0}\right),
\end{aligned}
$$

where $g_{i, k}:=g_{i, k}\left(\bar{x}_{i, k}, x_{i, \mu_{k}}\right)=\partial f_{i, k}\left(\bar{x}_{i, k}, x_{i, \mu_{k}}\right) / \partial x_{i, \mu_{k}} \quad$ with $k=1,2, \ldots, n_{i}, \quad x_{i, n_{i}+1}=u_{i}, \quad x_{i, \mu_{k}}=\mu_{k} x_{i, k+1}+\left(1-\mu_{k}\right) x_{i, k 0}$ with $0<\mu_{k}<1$, and $x_{i, k 0}$ are known at the given time $t_{0}$.

Therefore, system (1) can be rewritten as

$$
\left\{\begin{array}{l}
\dot{x}_{i, k}=f_{i, k}\left(\bar{x}_{i, k}(t), x_{i, k 0}\right)+g_{i, k} \cdot\left(x_{i, k+1}-x_{i, k 0}\right)+d_{i, k}\left(\bar{x}_{i, k}, t\right), \\
\dot{x}_{i, n_{i}}=f_{i, n_{i}}\left(\bar{x}_{i, n_{i}}(t), x_{i, n_{i}, 0}\right)+g_{i, n_{i}} \cdot\left(u_{i}-x_{i, n_{i}, 0}\right)+d_{i, n_{i}}\left(\bar{x}_{i, n_{i}}, t\right), \\
y_{i}=x_{i, 1}
\end{array}\right.
$$

Assumption 1 (see [37]). $g_{i, k}(\cdot)$ are unknown nonlinear functions and bounded, and there exists a positive constant $b_{i}$ such that $b_{i} \leq\left|g_{i, k}(\cdot)\right|$.

For ease of description, we defined vector functions $\bar{y}_{0, i}=\left[y_{0}, y_{0}^{(1)}, \ldots, y_{0}^{(i)}\right]^{T}$, where $y_{0}$ is the leader's output and $y_{0}^{(i)}$ is its $i^{\text {th }}$ derivative.

Assumption 2. Suppose that the leader's vector functions $\bar{y}_{0, i}$ are known smooth continuous functions and bounded. $\bar{y}_{0, i} \in \Omega_{0, i} \subset R^{i+1}$ with $i=1, \ldots, n_{i}$, where $\Omega_{0, i}$ being known compact sets and $y_{0}$ is an $n_{i}$-order differentiable function.

2.2. Graph Theory. The communication between agents can be represented by a directed graph $\mathscr{G}=(\mathscr{V}, \mathscr{E}, \mathscr{A})$, where $\mathscr{V}=\left\{N_{1}, N_{2}, \ldots, N_{M}\right\}$ denotes the set of nodes, and $\mathscr{E} \subseteq \mathscr{V} \times \mathscr{V}$ denotes the set of edges. An edge $(j, i) \in \mathscr{E}$ means that agent $i$ can obtain information from agent $j$, but not vice versa, where $j$ and $i$ are the parent node and child node, respectively. $\mathcal{N}_{i}=\{j \mid(j, i) \in \mathscr{E}\}$ is the set of neighbors of a node $i$, which is the set of nodes with edges incoming to node i. $\mathscr{A}=\left[a_{i, j}\right] \in \mathbb{R}^{M \times M}$ is the related adjacency matrix, if $(j, i) \in \mathscr{E}, a_{i j}>0$; otherwise, $a_{i, j}=0$. Self-edges are not allowed, i.e., $a_{i, j}=0$. The Laplacian matrix for graph $\mathscr{G}$ is denoted by $\mathscr{L}=\left(l_{i, j}\right)_{N \times N}$ with $l_{i, i}=\sum_{j=1, j \neq i}^{N}\left|a_{i, j}\right|$ and $l_{i, j}=$ $-a_{i, j}$ for $i \neq j$, and $\mathscr{L}=\mathscr{D}-\mathscr{A} \in \mathbb{R}^{M \times M}$ where 
$\mathscr{D}=\operatorname{diag}\left[d_{1}, \ldots, d_{M}\right] ; d_{i}=\sum_{j=1, j \neq i}^{M} a_{i j}$ is the diagonal element of the degree matrix $\mathscr{D}$. A directed graph $\mathscr{G}$ has a directed spanning tree if there exists at least one agent that has directed paths to all other agents.

If including a leader agent $N_{0}$, the communication topology for the $M+1$ agents is described by a directed graph $\overline{\mathscr{G}}=(\overline{\mathscr{V}}, \overline{\mathscr{E}}, \overline{\mathscr{A}})$, where $\overline{\mathscr{V}}=\mathscr{V} \cup\left\{N_{0}\right\}$ and $\overline{\mathscr{E}}=\overline{\mathscr{V}} \times \overline{\mathscr{V}}$. The connection matrix between the followers and the leader can be expressed as $H=\operatorname{diag}\left\{h_{1}, \ldots, h_{N}\right\}$ where $h_{i}>0$ if and only if $N_{i}$ can directly obtain the leader's information; otherwise, $h_{i}=0$.

2.3. RBF Neural Networks. RBF neural network [38] has powerful unknown function approximation ability. In this paper, we approximate uncertain nonlinear iterms in each agent's dynamics via the following RBF neural network:

$$
\hat{f}(Z)=W^{T} S(Z),
$$

where the input vector $Z \in \Omega_{Z} \subset R^{q}, W=\left[w_{1}, w_{2}, \ldots\right.$, $\left.w_{l}\right]^{T} \in R^{l}$ is the weight vector; $S(Z)=\left[s_{1}(Z), s_{2}(Z), \ldots\right.$, $\left.s_{l}(Z)\right]^{T} \in R^{l}$ is the radial basis function vector with neural network node number $l>0$. The basis function $S(Z)$ is always chosen as the following form of Gaussian function:

$$
S_{i}(Z)=\exp \left[-\frac{\left(Z-\mu_{i}\right)^{T}\left(Z-\mu_{i}\right)}{\eta_{i}^{2}}\right], \quad i=1,2, \ldots, l,
$$

where $\eta_{i}$ is the width of the Gaussian function, and $\mu_{i}=$ $\left[\mu_{i 1}, \mu_{i 2}, \ldots, \mu_{i q}\right]^{T}$ is the center of the receptive field. In theory, as long as a sufficient number $l$ of nodes are selected, RBF neural network can approximate any continuous function $f(Z)$ in a compact set $\Omega_{Z} \subset R^{q}$ with arbitrary precision $\varepsilon$.

$$
f(Z)=W^{* T} S(Z)+\delta(Z), \quad \forall Z \in \Omega_{Z} \in R^{q},
$$

where $\delta(Z)$ is the approximation error and satisfies $|\delta(Z)| \leq \varepsilon$, and $W^{*}$ is the ideal weight vector, which is chosen as the value of $W$ that minimizes $\varepsilon$ for all $Z \in \Omega_{Z}$, i.e.,

$$
W^{*}=\arg \min _{W \in R^{\prime}} \sup _{Z \in \Omega_{Z}}\{|f(Z)-\widehat{f}(Z)|\} .
$$

Remark 1. In this paper, we choose $\theta_{i, j}=\max \left\{\left\|W_{i, j}^{*}\right\| / b_{i}, i=\right.$ $\left.1,2, \ldots, M, j=1,2, \ldots, n_{i}\right\}$ with $\widetilde{\theta}_{i, j}=\theta_{i, j}-\widehat{\theta}_{i, j}$, where $\widehat{\theta}_{i, j}$ are the estimates of the unknown constants $\theta_{i, j}, b_{i}$ are positive design parameters and related to Assumption 1, and $\|\cdot\|$ is the norm.

Assumption 3 (see [39]). There exist unknown constants $Q_{i, j}$ such that $\left|\tilde{\theta}_{i, j}\right| \leq Q_{i, j}<\infty, i=1,2, \ldots, M, j=1,2, \ldots, n_{i}$.

Lemma 1 (see [40]). Consider the Gaussian function (5). $\|S(Z)\|$ has an upper bound such that $\|S(Z)\| \leq \sum_{k=0}^{\infty}$ $3 q(k+2)^{q^{-}} \quad 1 e^{-2 p^{2 k} k^{2} / \eta^{2}}:=s$, where $\quad p=(1 / 2)\left(\min _{i \neq j}\right.$ $\left.\left\|\mu_{i}-\mu_{j}\right\|\right)$.
2.4. Fixed-Time Control. Consider the following nonlinear system,

$$
\dot{x}(t)=f(x(t)), \quad x(0)=x_{0}, f(0)=0,
$$

where $x(t) \in R^{n}$ is a system variable, and $f(x): R^{n} \longrightarrow R^{n}$ is a nonlinear function which may be discontinuous; the solutions of system (8) are understood in the sense of Filippov [41]. For system (8), the following definitions and lemmas are introduced.

Definition 1 (see [1]). The equilibrium $x=0$ of system (8) is finite-time convergent if there are an open neighborhood $U \subseteq D$ of the origin and a positive definite function $T\left(x_{0}\right): U \backslash\{0\} \longrightarrow(0, \infty)$, such that $\lim _{t \longrightarrow T\left(x_{0}\right)} x\left(t, x_{0}\right)=0$ and $x\left(t, x_{0}\right)=0, \forall t>T\left(x_{0}\right)$ for any initial condition $x_{0} \in U$, where $T\left(x_{0}\right)$ is the settling time function. The equilibrium $x=0$ of system (8) is finite-time stable if it is Lyapunov stable and finite-time convergent. Moreover, when $U=D=R^{n}$, then the equilibrium $x=0$ is globally finitetime stable.

Definition 2 (see [19]). The equilibrium of system (8) is said to be fixed-time stable if it is globally finite-time stable and the settling time function $T\left(x_{0}\right)$ is uniformly bounded for any initial states, that is, $\exists T_{\max }$ such that $T\left(x_{0}\right) \leq T_{\max }$, $\forall x_{0} \in R^{n}$.

Lemma 2 (see [39]). Considering system (8), suppose that there exists a continuous differentiable positive definite function $V(x): R^{n} \longrightarrow R$ such that the following inequality holds:

$$
\dot{V}(x) \leq-\phi_{1} V^{\alpha}(x)-\phi_{2} V^{\beta}(x)+\tau,
$$

where $\phi_{1}>0, \phi_{2}>0, \alpha \in(0,1), \beta \in(1, \infty), \tau \in(0, \infty)$, and $\omega \in(0,1)$. Then, the equilibrium of system (8) is fixed-time stable and the convergence time function $T$ satisfies the following inequality:

$$
T \leq T_{\max }:=\frac{1}{\phi_{1} \oplus(1-\alpha)}+\frac{1}{\phi_{2} \bowtie(\beta-1)} .
$$

The residual set of the solution of system (8) is given by

$$
x \in\left\{V(x) \leq \min \left\{\left(\frac{\tau}{(1-\omega) \phi_{1}}\right)^{1 / \alpha},\left(\frac{\tau}{(1-\omega) \phi_{2}}\right)^{1 / \beta}\right\}\right\} \text {. }
$$

Lemma 3 (see [42]). For $x_{i} \in R, i=1,2, \ldots, n, 0<r_{1} \leq 1$, $r_{2}>1$, the following inequalities hold:

$$
\begin{aligned}
& \left(\sum_{i=1}^{n}\left|x_{i}\right|\right)^{r_{1}} \leq \sum_{i=1}^{n}\left|x_{i}\right|^{r_{1}}, \\
& \left(\sum_{i=1}^{n}\left|x_{i}\right|\right)^{r_{2}} \leq n^{r_{2}-1} \sum_{i=1}^{n}\left|x_{i}\right|^{r_{2}} .
\end{aligned}
$$

Lemma 4 (see [40]) (see [43]). For $x \in R$ and any positive constant $\xi$, the following inequality holds: 


$$
0 \leq|x|<\xi+\frac{x^{2}}{\sqrt{x^{2}+\xi^{2}}}
$$

Lemma 5. For $a \geq b>0, b, a \in R$ and any positive constant $c$, then the following inequality is satisfied:

$$
\frac{a}{\sqrt{a+c}} \geq \frac{b}{\sqrt{b+c}}
$$

Proof

$$
\frac{a}{\sqrt{a+c}}-\frac{b}{\sqrt{b+c}}=\frac{a+c}{\sqrt{a+c}}-\frac{b+c}{\sqrt{b+c}}-\frac{c}{\sqrt{a+c}}+\frac{c}{\sqrt{b+c}}=(\sqrt{a+c}-\sqrt{b+c})+\left(\frac{c}{\sqrt{b+c}}-\frac{c}{\sqrt{a+c}}\right) \geq 0 .
$$

2.5. Tracking Error Constraint. In this paper, we will investigate the output feedback tracking control problem with prescribed performance. The local tracking error for the $i$ th follower is defined as

$$
e_{i}=\sum_{j=1}^{M} a_{i j}\left(y_{i}-y_{j}\right)+h_{i}\left(y_{i}-y_{0}\right)
$$

To meet the requirement of output performance constraint, a positive decreasing smooth function as the following is selected:

$$
F_{b}=\xi_{0} e^{-a t}+\xi_{\infty}
$$

where $\xi_{0}>0, \xi_{\infty}>0$ are proper parameters, $a$ is a positive constant, and $\left|e_{i}(0)\right|<F_{b}(0)$.
Defining the following state transformation as the error constraint variable of the $i$ th follower,

$$
z_{i, 1}(t)=\frac{e_{i}(t)}{\sigma_{1} F_{b}-e_{i}} q\left(e_{i}(t)\right)+\frac{e_{i}(t)}{\sigma_{2} F_{b}+e_{i}}\left(1-q\left(e_{i}(t)\right)\right)
$$

where $\sigma_{1}$ and $\sigma_{2}$ are proper design parameters. $F_{b}$ satisfies the condition given in (17) and $q\left(e_{i}(t)\right)$ satisfies

$$
q\left(e_{i}(t)\right)= \begin{cases}1, & e_{i}(t) \geq 0 \\ 0, & e_{i}(t)<0\end{cases}
$$

The time derivative of $z_{i, 1}$ is

$$
\dot{z}_{i, 1}=\frac{1}{\left(\sigma_{1} F_{b}-e_{i}\right)^{2}}\left[\dot{e}_{i}\left(\sigma_{1} F_{b}-e_{i}\right)-e_{i}\left(\sigma_{1} \dot{F}_{b}-\dot{e}_{i}\right)\right] q+\frac{1}{\left(\sigma_{2} F_{b}+e_{i}\right)^{2}}\left[\dot{e}_{i}\left(\sigma_{2} F_{b}+e_{i}\right)-e_{i}\left(\sigma_{2} \dot{F}_{b}+\dot{e}_{i}\right)\right] \cdot(1-q)
$$

For ease of writing, we obtain

$$
\dot{z}_{i, 1}=F_{b} \Phi_{F_{i}} \dot{e}_{i}-\dot{F}_{b} \Phi_{F_{i}} e_{i}
$$

where

$$
\Phi_{F_{i}}=\frac{\sigma_{1} q}{\left(\sigma_{1} F_{b}-e_{i}\right)^{2}}+\frac{\sigma_{2}(1-q)}{\left(\sigma_{2} F_{b}+e_{i}\right)^{2}} .
$$

Remark 2. From the definition of $z_{i, 1}$ and $q\left(e_{i}(t)\right)$, it is obvious that when tracking error approaches the boundary $F_{b}, z_{i, 1}$ increases as a result. Then, by choosing proper parameters, the tracking error will be forced within prescribed boundary, i.e., $-\sigma_{2} F_{b}(t)<e_{i}(t)<\sigma_{1} F_{b}(t)$.

\section{Fixed-Time Controller Design}

In this section, an adaptive RBF neural network fixed-time controller is designed for MASs with uncertain nonlinear pure-feedback in the framework of backstepping method. The coordinate transformation is as follows:

$$
z_{i, k}=x_{i, k}-v_{i, k-1},
$$

where $i=1,2, \ldots, M, k=2,3, \ldots, n_{i}$, and $v_{i, k}$ represents the virtual controller of the $k$ th subsystem of the $i$ th follower, and $z_{i, 1}$ is given in (18)

In this paper, we employ $\mathrm{RBF}$ neural network to approximate uncertain functions $\widehat{f}_{i, k}\left(Z_{i, k}\right)$,

$$
\widehat{f}_{i, k}\left(Z_{i, k}\right)=W_{i, k}^{* T} S_{i, k}\left(Z_{i, k}\right)+\delta_{i, k}\left(Z_{i, k}\right) .
$$


From Young's inequality, complete square formula, and Lemma 3, the following inequalities hold:

$$
\begin{gathered}
z_{i, k} \delta_{i, k}\left(Z_{i, k}\right) \leq b_{i} \zeta_{i, k_{3}} z_{i, k}^{2}+\frac{\varepsilon_{i, k}^{2}}{4 b_{i} \zeta_{i, k_{3}}}, \\
z_{i, k} W_{i, k}^{* T} S_{i, k}\left(Z_{i, k}\right) \leq \frac{b_{i} \theta_{i, k}}{2 \eta_{i, k}^{2}} S_{i, k}^{T}\left(Z_{i, k}\right) S_{i, k}\left(Z_{i, k}\right) z_{i, k}^{2}+\frac{\eta_{i, k}^{2}}{2}, \\
-b_{i} \zeta_{i, k_{1}} 2^{(1+\alpha) / 2}\left(\left(\frac{1}{2} z_{i, k}^{2}\right)^{(1+\alpha) / 2}+\left(\frac{b_{i} \widetilde{\theta}_{i, k}^{2}}{2 \gamma_{i}}\right)^{(1+\alpha) / 2}\right) \leq-b_{i} \zeta_{i, k_{1}} 2^{(1+\alpha) / 2}\left(\frac{1}{2} z_{i, k}^{2}+\frac{b_{i} \widetilde{\theta}_{i, k}^{2}}{2 \gamma_{i}}\right)^{(1+\alpha) / 2}, \\
-b_{i} \zeta_{i, k_{2}} 2^{(1+\beta) / 2}\left(\left(\frac{1}{2} z_{i, k}^{2}\right)^{(1+\beta) / 2}+\left(\frac{b_{i} \widetilde{\theta}_{i, k}^{2}}{2 \gamma_{i}}\right)^{(1+\beta) / 2}\right)
\end{gathered}
$$

where $\quad Z_{i, k}=\left[x_{i, 1}, \ldots, x_{i, k}, \widehat{\theta}_{i, 1}, \ldots, \widehat{\theta}_{i, k}, h_{i} \bar{y}_{0, k}^{T}, a_{i 1} y_{1,1}, \ldots\right.$, $\left.a_{i M} y_{M, 1}, \ldots, a_{i 1} y_{1, k}, \ldots, a_{i M} y_{M, k}, F_{b}, \dot{F}_{b}, \ldots, F_{b}^{(k)}\right] \quad$ with $i=1, \ldots, M, k=1, \ldots, n_{i}$ is the input vector, and $b_{i}, \zeta_{i, k_{3}}$, $\zeta_{i, k_{2}}, \zeta_{i, k_{1}}, \varepsilon_{i, k}, \eta_{i, k}$, and $\gamma_{i}$ are positive parameters.

$$
V_{i, 1}=\frac{1}{2} z_{i, 1}^{2}+\frac{b_{i} \tilde{\theta}_{i, 1}^{2}}{2 \gamma_{i}}
$$

From (3), (16), and (18)-(23), we can obtain the time derivative of $V_{i, 1}$ :

Step 1. Choosing the Lyapunov function as

$$
\dot{V}_{i, 1}=\left(d_{i}+h_{i}\right) z_{i, 1} F_{b} \Phi_{F_{i}}\left(f_{i, 1}+g_{i, 1} z_{i, 2}+g_{i, 1} v_{i, 1}-g_{i, 1} x_{i, 10}+d_{i, 1}\right)-z_{i, 1} F_{b} \Phi_{F_{i}}\left(\sum_{j=1}^{M} a_{i j} \dot{x}_{j, 1}+h_{i} \dot{y}_{0}\right)-z_{i, 1} \dot{F}_{b} \Phi_{F_{i}} e_{i}-\frac{b_{i} \tilde{\theta}_{i, 1} \widehat{\theta}_{i, 1}}{\gamma_{i}}
$$

The virtual controller $v_{i, 1}$ is defined as

$$
v_{i, 1}=\frac{1}{\left(d_{i}+h_{i}\right) F_{b} \Phi_{F_{i}}}\left(-\frac{\widehat{\theta}_{i, 1}}{2 \eta_{i, 1}^{2}} S_{i, 1}^{T}\left(Z_{i, 1}\right) S_{i, 1}\left(Z_{i, 1}\right) z_{i, 1}-\zeta_{i, 11} S_{z_{i, 1}, 1}-\zeta_{i, 12} S_{z_{i, 1}, 2}-\zeta_{i, 13} z_{i, 1}+x_{i, 10}\right)
$$

where $\zeta_{i, 11}, \zeta_{i, 12}, \zeta_{i, 13}$, and $\eta_{i, 1}$ are positive design parameters.

$S_{z i, 1,1}$ and $S_{z i, 1,2}$ are defined by the following equalities: 


$$
\begin{aligned}
S_{z_{i, k}, 1} & = \begin{cases}\frac{\left(z_{i, k}^{2}\right)^{(1+\alpha) / 2}}{z_{i, k}}, & \left|z_{i, k}\right| \geq \varepsilon_{k 0}, \\
\sum_{j=1}^{n_{i}} a_{j}\left(z_{i, k}^{2}\right)^{j}\left(\varepsilon_{k 0}^{2}\right)^{-j+((1+\alpha) / 2)}, & \left|z_{i, k}\right|<\varepsilon_{k 0},\end{cases} \\
S_{z_{i, k}, 2} & = \begin{cases}\frac{\left(z_{i, k}^{2}\right)^{(1+\beta) / 2}}{z_{i, k}}, & \left|z_{i, k}\right| \geq \varepsilon_{k 0}, \\
\sum_{j=1}^{n_{i}} a_{j}\left(z_{i, k}^{2}\right)^{j}\left(\varepsilon_{k 0}^{2}\right)^{-j+((1+\beta) / 2)}, & \left|z_{i, k}\right|<\varepsilon_{k 0},\end{cases}
\end{aligned}
$$

where $k=1, \ldots, n_{i}, \varepsilon_{k 0}$ is the positive design parameter, and coefficients $a_{j}$ with $j=1, \ldots, n_{i}$ are calculated using the following equation:

$$
\left[\begin{array}{ccccc}
1 & 1 & \cdots & 1 & 1 \\
1 & 2 & \cdots & n_{i}-1 & n_{i} \\
0 & 2 \times 1 & \cdots & \left(n_{i}-1\right)\left(n_{i}-2\right) & n_{i}\left(n_{i}-1\right) \\
\vdots & \vdots & \ddots & \vdots & \vdots \\
0 & 0 & \cdots & \prod_{j=0}^{n_{i}-2}\left(n_{i}-1-j\right) & \prod_{j=0}^{n_{i}-2}\left(n_{i}-j\right)
\end{array}\right] \times\left[\begin{array}{c}
a_{1} \\
a_{2} \\
a_{3} \\
\vdots \\
a_{n_{i}}
\end{array}\right]=\left[\begin{array}{c}
b_{1} \\
b_{2} \\
b_{3} \\
\vdots \\
b_{n_{i}}
\end{array}\right]
$$

where $b_{1}=1, \quad b_{2}=3 / 4, \quad b_{3}=(3 / 4)((3 / 4)-1), \ldots$, and $b_{n}=\prod_{j=0}^{n_{i}-2}((3 / 4)-j)$.

Remark 3. The above process is to eliminate the problem of fixed-time controller's singularity. In this paper, we just consider the situation of $\left|z_{i, k}\right| \geq \varepsilon_{k 0}$, since when $\left|z_{i, k}\right|<\varepsilon_{k 0}$, there we just add an additional term and it clearly does not affect the final conclusion.

Substituting (31) into (30) yields

$$
\begin{aligned}
\dot{V}_{i, 1} \leq & \left(d_{i}+h_{i}\right) z_{i, 1} F_{b} \Phi_{F_{i}} g_{i, 1} z_{i, 2}+z_{i, 1} \widehat{f}_{i, 1}\left(Z_{i, 1}\right)-\frac{b_{i} \widehat{\theta}_{i, 1}^{2}}{2 \eta_{i, 1}^{2}} S_{i, 1}^{T}\left(Z_{i, 1}\right) S_{i, 1}\left(Z_{i, 1}\right) z_{i, 1}^{2}-b_{i} \zeta_{i, 11} z_{i, 1} S_{z_{i, 1}, 1}-b_{i} \zeta_{i, 12} z_{i, 1} S_{z_{i, 1}, 2} \\
& -b_{i} \zeta_{i, 13} z_{i, 1}^{2}-\frac{b_{i} \widetilde{\theta}_{i, 1} \dot{\hat{\theta}}_{i, 1}}{\gamma_{i}}
\end{aligned}
$$

where $\hat{f}_{i, 1}\left(Z_{i, 1}\right)=\left(d_{i}+h_{i}\right) F_{b} \Phi_{F_{i}}\left(f_{i, 1}+d_{i, 1}\right)-F_{b} \Phi_{F_{i}}\left(\sum_{j=1}^{M}\right.$

From (24)-(26), we have $\left.a_{i j} \dot{x}_{j, 1}+h_{i} \dot{y}_{0}\right)-\dot{F}_{b} \Phi_{F_{i}} e_{i}$.

$$
\dot{V}_{i, 1} \leq \frac{b_{i} \tilde{\theta}_{i, 1}}{\gamma_{i}}\left(\frac{\gamma_{i}}{2 \eta_{i, 1}^{2}} S_{i, 1}^{T}\left(Z_{i, 1}\right) S_{i, 1}\left(Z_{i, 1}\right) z_{i, 1}^{2}-\dot{\widehat{\theta}}_{i, 1}\right)+\left(d_{i}+h_{i}\right) z_{i, 1} F_{b} \Phi_{F_{i}} g_{i, 1} z_{i, 2}+\sigma_{i, 1}-b_{i} \zeta_{i, 11} z_{i, 1} S_{z_{i, 1}, 1}-b_{i} \zeta_{i, 12} z_{i, 1} S_{z_{i, 1}, 2}
$$

where $\sigma_{i, 1}=(1 / 2) \eta_{i, 1}^{2}+\left(\varepsilon_{i, 1}^{2} / 4 b_{i} \zeta_{i, 13}\right)$.

The adaptive law $\hat{\theta}_{i, 1}$ is defined as

$$
\dot{\hat{\theta}}_{i, 1}=\frac{\gamma_{i}}{2 \eta_{i, 1}^{2}} S_{i, 1}^{T}\left(Z_{i, 1}\right) S_{i, 1}\left(Z_{i, 1}\right) z_{i, 1}^{2}-\lambda_{i} \widehat{\theta}_{i, 1}
$$


where $\lambda_{i}$ with $i=1, \ldots, M$ is the positive parameter.

From Assumption 3 and (37), we have

$$
\begin{aligned}
\dot{V}_{i, 1} \leq & -b_{i} \zeta_{i, 11} z_{i, 1} S_{z_{i, 1}, 1}-b_{i} \zeta_{i, 12} z_{i, 1} S_{z_{i, 1}, 2}-b_{i} \zeta_{i, 11} 2^{(1+\alpha) / 2}\left(\frac{b_{i} \widetilde{\theta}_{i, 1}^{2}}{2 \gamma_{i}}\right)^{(1+\alpha) / 2}-b_{i} \zeta_{i, 12} 2^{(1+\beta) / 2}\left(\frac{b_{i} \widetilde{\theta}_{i, 1}^{2}}{2 \gamma_{i}}\right)^{(1+\beta) / 2} \\
& +\left(d_{i}+h_{i}\right) z_{i, 1} F_{b} \Phi_{F_{i}} g_{i, 1} z_{i, 2}+C_{i, 1},
\end{aligned}
$$

where $\quad C_{i, 1}=\sigma_{i, 1}+\beta_{i, 1}+\left(\lambda_{i} b_{i} / 2 \gamma_{i}\right) \theta_{i, 1}^{2}, \quad \beta_{i, 1}=\zeta_{i, 11} 2^{(1+\alpha) / 2}$ $\left(b_{i} Q_{i, 1}^{2} / 2 \gamma_{i}\right)^{(1+\alpha) / 2}+\zeta_{i, 12} 2^{(1+\beta) / 2}\left(b_{i} Q_{i, 1}^{2} / 2 \gamma_{i}\right)^{(1+\beta) / 2}$, and $\left(\lambda_{1} b_{i} \quad\left|z_{i, 1} \geq \varepsilon_{10}\right|\right.$,

According to (27), (28), (32), and (33), and when $\left.\widetilde{\theta}_{i, 1} \widehat{\theta}_{i, 1} / \gamma_{i}\right) \leq\left(\lambda_{i} b_{i} / \gamma_{i}\right)\left(\left(\theta_{i, 1}^{2} / 2\right)-\left(\tilde{\theta}_{i, 1}^{2} / 2\right)\right) \leq\left(\lambda_{i} b_{i} / 2 \gamma_{i}\right) \theta_{i, 1}^{2}$.

$$
\dot{V}_{i, 1} \leq-b_{i} \zeta_{i, 11} 2^{(1+\alpha) / 2}\left(\frac{1}{2} z_{i, 1}^{2}+\frac{b_{i} \tilde{\theta}_{i, 1}^{2}}{2 \gamma_{i}}\right)^{(1+\alpha) / 2}-2 b_{i} \zeta_{i, 12}\left(\frac{1}{2} z_{i, 1}^{2}+\frac{b_{i} \widetilde{\theta}_{i, 1}^{2}}{2 \gamma_{i}}\right)^{(1+\beta) / 2}+\left(d_{i}+h_{i}\right) z_{i, 1} F_{b} \Phi_{F_{i}} g_{i, 1} z_{i, 2}+C_{i, 1}
$$

Step 2. From (3) and (23), we can obtain

$$
\begin{aligned}
\dot{z}_{i, 2}= & \dot{x}_{i, 2}-\dot{v}_{i, 1} \\
= & f_{i, 2}\left(\bar{x}_{i, 2}, x_{i, 20}\right)+g_{i, 2}\left(x_{i, 3}-x_{i, 20}\right) \\
& +d_{i, 2}-\dot{v}_{i, 1},
\end{aligned}
$$

Construct Lyapunov function as

$$
V_{i, 2}=V_{i, 1}+\frac{1}{2} z_{i, 2}^{2}+\frac{b_{i} \tilde{\theta}_{i, 2}^{2}}{2 \gamma_{i}}
$$

The derivative of $V_{i, 2}$ is written as

where $\quad \dot{v}_{i, 1}=\left(\partial v_{i_{1}} / \partial x_{i_{1}, 1}\right) \dot{x}_{i_{i, 1}}+\sum_{j=1}^{M} a_{i j}\left(\left(\partial v_{i_{1,1}} / \partial x_{j_{j}}\right) x_{j, 1}+\right.$ $\left.\left(\partial v_{i, 1} / \partial x_{j, 2}\right) \dot{x}_{j, 2}\right)+h_{i}\left(\left(\partial v_{i, 1} / \partial x_{0,1}\right) \dot{x}_{0,1}+\left(\partial v_{i, 1} / \partial x_{0,2}\right) \dot{x}_{0,2}\right)+$ $\left(\partial v_{i, 1} / \partial \widehat{\theta}_{i, 1}\right) \dot{\hat{\theta}}_{i, 1}+\left(\partial v_{i, 1} / \partial F_{b}\right) \dot{F}_{b}+\left(\partial v_{i, 1} / \partial \vec{F}_{b}\right) \ddot{F}_{b}$.

$$
\begin{aligned}
& \dot{V}_{i, 2} \leq-b_{i} \zeta_{i, 11} 2^{(1+\alpha) / 2}\left(\frac{1}{2} z_{i, 1}^{2}+\frac{b_{i} \widetilde{\theta}_{i, 1}^{2}}{2 \gamma_{i}}\right)^{(1+\alpha) / 2}+z_{i, 2} \widehat{f}_{i, 2}\left(Z_{i, 2}\right)-2 b_{i} \zeta_{i, 12}\left(\frac{1}{2} z_{i, 1}^{2}+\frac{b_{i} \widetilde{\theta}_{i, 1}^{2}}{2 \gamma_{i}}\right)^{(1+\beta) / 2} \\
& +z_{i, 2} g_{i, 2} z_{i, 3}+z_{i, 2} g_{i, 2} v_{i, 2}-z_{i, 2} g_{i, 2} x_{i, 20}-z_{i, 2} \frac{\partial v_{i, 1} \hat{\widehat{\theta}}_{i, 1}}{\partial \hat{\theta}_{i, 1}}-\frac{b_{i} \widetilde{\theta}_{i, 2} \dot{\hat{\theta}}_{i, 2}}{\gamma_{i}}+z_{i, 2} M_{i, 1}\left(Z_{i, 2}\right)+C_{i, 1},
\end{aligned}
$$

where $\quad \hat{f}_{i, 2}\left(Z_{i, 2}\right)=f_{i, 2}\left(\bar{x}_{i, 2}, x_{i, 20}\right)+d_{i, 2}+\left(d_{i}+h_{i}\right) z_{i, 1} F_{b}$ $\Phi_{F_{i}} g_{i, 1}-\left(\partial v_{i_{1,1}} / \partial x_{i, 1}\right) \dot{x}_{i, 1}-\sum_{j=1}^{M} a_{i j}\left(\left(\partial v_{i, 1} / \partial x_{j, 1}\right) \dot{x}_{j, 1}+\left(\partial v_{i, 1} /\right.\right.$ $\left.\left.\partial x_{j, 2}\right) \dot{x}_{j, 2}\right)-h_{i}\left(\left(\partial v_{i, 1} / \partial x_{0,1}\right) \dot{x}_{0,1}+\left(\partial v_{i, 1} / \partial x_{0,2}\right) \dot{x}_{0,2}\right)-M_{i, 1}$ $\left(Z_{i, 2}\right)$.
Remark 4. $M_{i, 1}\left(Z_{i, 2}\right)$ is a smooth function that is used to compensate $\left(\partial v_{i, 1} / \partial \hat{\theta}_{i 1}\right) \dot{\hat{\theta}}_{i, 1}$.

The virtual controller $v_{i, 2}$ is defined as

$$
v_{i, 2}=-\frac{\hat{\theta}_{i, 2}}{2 \eta_{i, 2}^{2}} S_{i, 2}^{T}\left(Z_{i, 2}\right) S_{i, 2}\left(Z_{i, 2}\right) z_{i, 2}+x_{i, 20}-\zeta_{i, 21} S_{z_{i, 2}, 1}-\zeta_{i, 22} S_{z_{i, 2}, 2}-\zeta_{i, 23} z_{i, 2}
$$

where $\zeta_{i, 21}, \zeta_{i, 22}, \zeta_{i, 23}$, and $\eta_{i, 2}$ are positive design parameters.

According to (24)-(26) and substituting (43) into (42), we have 


$$
\begin{aligned}
\dot{V}_{i, 2} \leq & \frac{b_{i} \tilde{\theta}_{i, 2}}{\gamma_{i}}\left(\frac{\gamma_{i}}{2 \eta_{i, 2}^{2}} S_{i, 2}^{T}\left(Z_{i, 2}\right) S_{i, 2}\left(Z_{i, 2}\right) z_{i, 2}^{2}-\dot{\hat{\theta}}_{i, 2}\right)+z_{i, 2} g_{i, 2} z_{i, 3}+\sigma_{i, 2}-b_{i} \zeta_{i, 21} z_{i, 2} S_{z_{i, 2}, 1}-b_{i} \zeta_{i, 22} z_{i, 2} S_{z_{i, 2}, 2}+C_{i, 1} \\
& -b_{i} \zeta_{i, 11} 2^{(1+\alpha) / 2}\left(\frac{1}{2} z_{i, 1}^{2}+\frac{b_{i} \widetilde{\theta}_{i, 1}^{2}}{2 \gamma_{i}}\right)^{(1+\alpha) / 2}-2 b_{i} \zeta_{i, 12}\left(\frac{1}{2} z_{i, 1}^{2}+\frac{b_{i} \widetilde{\theta}_{i, 1}^{2}}{2 \gamma_{i}}\right)^{(1+\beta) / 2}+z_{i, 2}\left(M_{i, 1}\left(Z_{i, 2}\right)-\frac{\partial v_{i, 1}}{\partial \widetilde{\theta}_{i, 1}} \dot{\theta}_{i, 1}\right),
\end{aligned}
$$

where $\sigma_{i, 2}=(1 / 2) \eta_{i, 2}^{2}+\left(\varepsilon_{i, 2}^{2} / 4 b_{i} \zeta_{i, 23}\right)$.

From Assumption 3 and (45), we have

Then, the adaptive law $\hat{\theta}_{i, 2}$ is defined as

$$
\dot{\hat{\theta}}_{i, 2}=\frac{\gamma_{i}}{2 \eta_{i, 2}^{2}} S_{i, 2}^{T}\left(Z_{i, 2}\right) S_{i, 2}\left(Z_{i, 2}\right) z_{i, 2}^{2}-\lambda_{i} \widehat{\theta}_{i, 2}
$$

$$
\begin{aligned}
\dot{V}_{i, 2} \leq & -b_{i} \zeta_{i, 21} z_{i, 2} S_{z_{i, 2}, 1}-b_{i} \zeta_{i, 22} z_{i, 2} S_{z_{i, 2}, 2}-b_{i} \zeta_{i, 21} 2^{(1+\alpha) / 2}\left(\frac{b_{i} \widetilde{\theta}_{i, 2}^{2}}{2 \gamma_{i}}\right)^{(1+\alpha) / 2} \\
& -b_{i} \zeta_{i, 22} 2^{(1+\beta) / 2}\left(\frac{b_{i} \widetilde{\theta}_{i, 2}^{2}}{2 \gamma_{i}}\right)^{(1+\beta) / 2}-b_{i} \zeta_{i, 11} 2^{(1+\alpha) / 2}\left(\frac{1}{2} z_{i, 1}^{2}+\frac{b_{i} \widetilde{\theta}_{i, 1}^{2}}{2 \gamma_{i}}\right)^{(1+\alpha) / 2} \\
& -2 b_{i} \zeta_{i, 12}\left(\frac{1}{2} z_{i, 1}^{2}+\frac{b_{i} \widetilde{\theta}_{i, 1}^{2}}{2 \gamma_{i}}\right)^{(1+\beta) / 2}+\sum_{j=1}^{2} C_{i, j}+z_{i, 2} g_{i, 2} z_{i, 3}+z_{i, 2}\left(M_{i, 1}\left(Z_{i, 2}\right)-\frac{\partial v_{i_{i, 1}} \dot{\hat{\theta}}_{i, 1}}{\partial \widehat{\theta}_{i, 1}}\right),
\end{aligned}
$$

where $C_{i, 2}=\sigma_{i, 2}+\beta_{i, 2}+\left(\lambda_{i} b_{i} / 2 \gamma_{i}\right) \theta_{i, 2}^{2}, \quad \beta_{i, 2}=b_{i} \zeta_{i, 21} 2^{(1+\alpha) / 2}$ $\left(b_{i} Q_{i, 2}^{2} / 2 \gamma_{i}\right)^{(1+\alpha) / 2}+b_{i} \zeta_{i, 22} 2^{(1+\beta) /} \quad 2\left(b_{i} Q_{i, 2}^{2} / 2 \gamma_{i}\right)^{(1+\beta) / 2}$, and $\left|z_{i, 2}\right| \geq \varepsilon_{20}$,

According to (27), (28), (32), and (33) and when $\left(\lambda_{1} b_{i} \tilde{\theta}_{i, 2} \widehat{\theta}_{i, 2} / \gamma_{i}\right) \leq\left(\lambda_{i} b_{i} / \gamma_{i}\right)\left(\left(\theta_{i, 2}^{2} / 2\right)-\left(\tilde{\theta}_{i, 2}^{2} / 2\right)\right) \leq\left(\lambda_{i} b_{i} / 2 \gamma_{i}\right) \theta_{i, 2}^{2}$

$$
\begin{aligned}
\dot{V}_{i, 2} \leq & -b_{i} 2^{(1+\alpha) / 2} \sum_{j=1}^{2} \zeta_{i, j 1}\left(\frac{1}{2} z_{i, j}^{2}+\frac{b_{i} \widetilde{\theta}_{i, j}^{2}}{2 \gamma_{i}}\right)^{(1+\alpha) / 2}-2 b_{i} \sum_{j=1}^{2} \zeta_{i, j 2}\left(\frac{1}{2} z_{i, j}^{2}+\frac{b_{i} \widetilde{\theta}_{i, j}^{2}}{2 \gamma_{i}}\right)^{(1+\beta) / 2}+z_{i, 2}\left(M_{i, 1}\left(Z_{i, 2}\right)-\frac{\partial v_{i, 1}}{\partial \widehat{\theta}_{i, 1}} \dot{\theta}_{i, 1}\right) \\
& +\sum_{j=1}^{2} C_{j}+z_{i, 2} g_{i, 2} z_{i, 3} .
\end{aligned}
$$

From Lemmas 1, 4, and 5 and (37), we have

$$
\begin{aligned}
-z_{i, 2} \frac{\partial v_{i, 1} \dot{\hat{\theta}}_{i, 1}}{\partial \widehat{\theta}_{i, 1}} & \leq-z_{i, 2} \frac{\partial v_{i, 1}}{\partial \widehat{\theta}_{i, 1}}\left(\frac{\gamma_{i}}{2 \eta_{i, 1}^{2}} S_{i, 1}^{T}\left(Z_{i, 1}\right) S_{i, 1}\left(Z_{i, 1}\right) z_{i, 1}^{2}-\lambda_{i} \widehat{\theta}_{i, 1}\right) \\
& \leq \frac{z_{i, 2}^{2}\left(\partial v_{i, 1} / \partial \widehat{\theta}_{i, 1}\right)^{2}\left(\left(\gamma_{i} / 2 \eta_{i, 1}^{2}\right) S_{i, 1}^{T}\left(Z_{i, 1}\right) S_{i, 1}\left(Z_{i, 1}\right) z_{i, 1}^{2}\right)^{2}}{\sqrt{z_{i, 2}^{2}\left(\partial v_{i, 1} / \partial \hat{\theta}_{i, 1}\right)^{2}\left(\left(\gamma_{i} / 2 \eta_{i, 1}^{2}\right) S_{i, 1}^{T}\left(Z_{i, 1}\right) S_{i, 1}\left(Z_{i, 1}\right) z_{i, 1}^{2}\right)^{2}+\kappa^{2}}}+z_{i, 2} \frac{\partial v_{i, 1}}{\partial \widehat{\theta}_{i, 1}} \lambda_{i} \widehat{\theta}_{i, 1} \\
& \leq \frac{z_{i, 2}^{2}\left(\partial v_{i, 1} / \partial \hat{\theta}_{i, 1}^{2}\right)^{2}\left(\left(\gamma_{i} / 2 \eta_{i, 1}^{2}\right) S^{2} z_{i, 1}^{2}\right)^{2}}{\sqrt{z_{i, 2}^{2}\left(\partial v_{i, 1} / \partial \widehat{\theta}_{i, 1}\right)^{2}\left(\left(\gamma_{i} / 2 \eta_{i, 1}^{2}\right) S^{2} z_{i, 1}^{2}\right)^{2}+\kappa^{2}}}+z_{i, 2} \frac{\partial v_{i, 1}}{\partial \widehat{\theta}_{i, 1}} \lambda_{i} \widehat{\theta}_{i, 1} .
\end{aligned}
$$


Therefore, $M_{i, 1}\left(Z_{i, 2}\right)$ can be defined as

$$
\begin{aligned}
M_{i, 1}\left(Z_{i, 2}\right)= & -\frac{z_{i, 2}\left(\partial v_{i, 1} / \partial \widehat{\theta}_{i, 1}\right)^{2}\left(\left(\gamma_{i} / 2 \eta_{i, 1}^{2}\right) S^{2} z_{i, 1}^{2}\right)^{2}}{\sqrt{z_{i, 2}^{2}\left(\partial v_{i, 1} / \partial \widehat{\theta}_{i, 1}\right)^{2}\left(\left(\gamma_{i} / 2 \eta_{i, 1}^{2}\right) S^{2} z_{i, 1}^{2}\right)^{2}+\kappa^{2}}} \\
& -\frac{\partial v_{i, 1}}{\partial \widehat{\theta}_{i, 1}} \widehat{\lambda}_{i} \widehat{\theta}_{i, 1},
\end{aligned}
$$

with the result that

$$
z_{i, 2}\left(M_{i, 1}\left(Z_{i, 2}\right)-\frac{\partial v_{i, 1}}{\partial \widehat{\theta}_{i, 1}^{i}} \widehat{\theta}_{i, 1}\right) \leq 0
$$

Substituting (50) into (47), we can obtain

$$
\dot{V}_{i, 2} \leq-b_{i} 2^{(1+\alpha) / 2} \sum_{j=1}^{2} \zeta_{i, j 1}\left(\frac{1}{2} z_{i, j}^{2}+\frac{b_{i} \widetilde{\theta}_{i, j}^{2}}{2 \gamma_{i}}\right)^{(1+\alpha) / 2}-2 b_{i} \sum_{j=1}^{2} \zeta_{i, j 2}\left(\frac{1}{2} z_{i, j}^{2}+\frac{b_{i} \widetilde{\theta}_{i, j}^{2}}{2 \gamma_{i}}\right)^{(1+\beta) / 2}+\sum_{j=1}^{2} C_{j}+z_{i, 2} g_{i, 2} z_{i, 3} .
$$

Step k. $\left(3 \leq k \leq n_{i}-1\right)$ : from (3) and (23), we have

$$
\begin{aligned}
\dot{z}_{i, k}= & \dot{x}_{i, k}-\dot{v}_{i, k-1} \\
= & f_{i, k}\left(\bar{x}_{i, k}, x_{i, k 0}\right)+g_{i, k}\left(x_{i, k+1}-x_{i, k 0}\right) \\
& +d_{i, k}-\dot{v}_{i, k-1},
\end{aligned}
$$

where $\dot{v}_{i, k-1}=\sum_{j=1}^{k-1}\left(\partial v_{i, k-1} / \partial x_{i, j}\right) \dot{x}_{i, j}+h_{i} \sum_{j=1}^{k}\left(\partial v_{i, k-1} /\right.$

$\left.\partial x_{0, j}\right) \dot{x}_{0, j}+\sum_{j=1}^{M} a_{i j} \sum_{q=1}^{k}\left(\left(\partial v_{i, k-1} / \partial x_{j, q}\right) \dot{x}_{j, q}\right)+\sum_{j=1}^{k-1}$

$\left(\partial v_{i, k-1} / \partial \hat{\theta}_{i, j}\right) \dot{\hat{\theta}}_{i, j}+\backslash \sum_{j=0}^{k-1}\left(\partial v_{i, k-1} / \partial F_{b}^{(j)}\right) F_{b}^{(j+1)}$.
Construct Lyapunov function as

$$
V_{i, k}=V_{i, k-1}+\frac{1}{2} z_{i, k}^{2}+\frac{b_{i} \widetilde{\theta}_{i, k}^{2}}{2 \gamma_{i}} .
$$

The derivative of $V_{i, k}$ is

$$
\begin{aligned}
& \dot{V}_{i, k} \leq-b_{i} 2^{(1+\alpha) / 2} \sum_{j=1}^{k-1} \zeta_{i, j 1}\left(\frac{1}{2} z_{i, j}^{2}+\frac{b_{i} \widetilde{\theta}_{i, j}^{2}}{2 \gamma_{i}}\right)^{(1+\alpha) / 2}-2 b_{i} \sum_{j=1}^{k-1} \zeta_{i, j 2}\left(\frac{1}{2} z_{i, j}^{2}+\frac{b_{i} \widetilde{\theta}_{i, j}^{2}}{2 \gamma_{i}}\right)^{(1+\beta) / 2} \\
& +z_{i, k} \widehat{f}_{i, k}\left(Z_{i, k}\right)+z_{i, k} g_{i, k} z_{i, k+1}+z_{i, k} g_{i, k} v_{i, k}-z_{i, k} g_{i, k} x_{i, k 0}-z_{i, k} \sum_{j=1}^{k-1} \frac{\partial v_{i, k-1}}{\partial \widehat{\hat{\theta}}_{i, j}}{ }_{i, j}-\frac{b_{i} \widetilde{\theta}_{i, k} \dot{\hat{\theta}}_{i, k}}{\gamma_{i}}+z_{i, k} M_{i, k-1}\left(Z_{i, k}\right)+\sum_{j=1}^{k-1} C_{i, j}
\end{aligned}
$$

where $\widehat{f}_{i, k}\left(Z_{i, k}\right)=f_{i, k}\left(\bar{x}_{i, k}, x_{i, k 0}\right)+d_{i, k}+z_{i, k-1} g_{i, k-1}-\sum_{j=1}^{k-1}$ $\left(\partial v_{i, k-1} / \partial x_{i, j}\right) \dot{x}_{i, j}-\sum_{j=1}^{M} a_{i j} \sum_{q=1}^{k}\left(\left(\partial v_{i, k-1} / \partial x_{j, q}\right) \dot{x}_{j, q}\right)-h_{i} \sum_{j=1}^{k}$ $\left(\partial v_{i, k-1} / \partial x_{0, j}\right) \dot{x}_{0, j}-\sum_{j=0}^{k-1}\left(\partial v_{i, k-1} / \partial F_{b}^{(j)}\right) F_{b}^{(j+1)}-M_{i, k-1}\left(Z_{i, k}\right)$.

The virtual controller $v_{i, k}$ is defined as

$$
\begin{aligned}
v_{i, k}= & -\frac{\hat{\theta}_{i, k}}{2 \eta_{i, k}^{2}} S_{i, k}^{T}\left(Z_{i, k}\right) S_{i, k}\left(Z_{i, k}\right) z_{i, k}+x_{i, k 0} \\
& -\zeta_{i, k 1} S_{z_{i, k}, 1}-\zeta_{i, k 2} S_{z_{i, k}, 2}-\zeta_{i, k 3} z_{i, k}
\end{aligned}
$$


where $\zeta_{i, k 1}, \zeta_{i, k 2}, \zeta_{i, k 3}$, and $\eta_{i, k}$ are positive design parameters.

According to 24)-(26) and substituting (55) into (54), we have

$$
\begin{aligned}
\dot{V}_{i, k} \leq & \frac{b_{i} \tilde{\theta}_{i, k}}{\gamma_{i}}\left(\frac{\gamma_{i}}{2 \eta_{i, k}^{2}} S_{i, k}^{T}\left(Z_{i, k}\right) S_{i, k}\left(Z_{i, k}\right) z_{i, k}^{2}-\widehat{\theta}_{i, k}\right)+z_{i, k} g_{i, k} z_{i, k+1}+\sigma_{i, k}-b_{i} \zeta_{i, k 1} z_{i, k} S_{z_{i, k}, 1}-b_{i} \zeta_{i, k 2} z_{i, k} S_{z_{i, k}, 2}+\sum_{j=1}^{k-1} C_{i, j} \\
& +z_{i, k}\left(M_{i, k-1}\left(Z_{i, k}\right)-\sum_{j=1}^{k-1} \frac{\partial v_{i, k-1}}{\partial \widehat{\theta}_{i, j}}\right)-b_{i} 2^{(1+\alpha) / 2} \sum_{j=1}^{k-1} \zeta_{i, j 1}\left(\frac{1}{2} z_{i, j}^{2}+\frac{b_{i} \widetilde{\theta}_{i, j}^{2}}{2 \gamma_{i}}\right)^{(1+\alpha) / 2}-2 b_{i} \sum_{j=1}^{k-1} \zeta_{i, j 2}\left(\frac{1}{2} z_{i, j}^{2}+\frac{b_{i} \widetilde{\theta}_{i, j}^{2}}{2 \gamma_{i}}\right)^{(1+\beta) / 2},
\end{aligned}
$$

where $\sigma_{i, k}=(1 / 2) \eta_{i, k}^{2}+\left(\varepsilon_{i, k}^{2} / 4 b_{i} \zeta_{i, k 3}\right)$.

Substituting (57) into (56), we can obtain

Then, the adaptive law $\widehat{\theta}_{i, k}$ is defined as

$$
\dot{\hat{\theta}}_{i, k}=\frac{\gamma_{i}}{2 \eta_{i, k}^{2}} S_{i, k}^{T}\left(Z_{i, k}\right) S_{i, k}\left(Z_{i, k}\right) z_{i, k}^{2}-\lambda_{i} \widehat{\theta}_{i, k} .
$$

$$
\begin{aligned}
\dot{V}_{i, k} \leq & -b_{i} 2^{(1+\alpha) / 2} \sum_{j=1}^{k-1} \zeta_{i, j 1}\left(\frac{1}{2} z_{i, j}^{2}+\frac{b_{i} \widetilde{\theta}_{i, j}^{2}}{2 \gamma_{i}}\right)^{(1+\alpha) / 2}-2 b_{i} \sum_{j=1}^{k-1} \zeta_{i, j 2}\left(\frac{1}{2} z_{i, j}^{2}+\frac{b_{i} \widetilde{\theta}_{i, j}^{2}}{2 \gamma_{i}}\right)^{(1+\beta) / 2}-b_{i} \zeta_{i, k 1} z_{i, k} S_{z_{i, k}, 1}-b_{i} \zeta_{i, k 2} z_{i, k} S_{z_{i, k}, 2} \\
& -b_{i} \zeta_{i, k 1} 2^{(1+\alpha) / 2}\left(\frac{b_{i} \widetilde{\theta}_{i, k}^{2}}{2 \gamma_{i}}\right)^{(1+\alpha) / 2}-b_{i} \zeta_{i, k 2} 2^{(1+\beta) / 2}\left(\frac{b_{i} \widetilde{\theta}_{i, k}^{2}}{2 \gamma_{i}}\right)^{(1+\beta) / 2}+z_{i, k}\left(M_{i, k-1}\left(Z_{i, k}\right)-\sum_{j=1}^{k-1} \frac{\left.\partial v_{i, k-1} \dot{\hat{\theta}}_{i, j}\right)+z_{i, k} g_{i, k} z_{i, k+1}+\sum_{j=1}^{k} C_{i, j}}{\partial,}\right.
\end{aligned}
$$

where $\quad C_{i, k}=\sigma_{i, k}+\beta_{i, k}+\left(\lambda_{i} b_{i} / 2 \gamma_{i}\right) \theta_{i, k}^{2}, \quad \beta_{l, k}=b_{i} \zeta_{i, k 1} 2^{(1+\alpha) / 2}$ According to (27), (28), (32), and (33) and when $\left(b_{i} Q_{l, k}^{2} / 2 \gamma_{i}\right)^{(1+\alpha) / 2}+b_{i} \zeta_{l, k 2} 2^{(1+\beta) / 2}\left(b_{i} Q_{i, k}^{2} / 2 \gamma_{i}\right)^{(1+\beta) / 2}$, and $\left(\lambda_{i} b_{i} \quad\left|z_{i, k} \geq \varepsilon_{k 0}\right|\right.$, $\left.\tilde{\theta}_{i, k} \widehat{\theta}_{i, k} / \gamma_{i}\right) \leq\left(\lambda_{i} b_{i} / \gamma_{i}\right)\left(\left(\theta_{i, k}^{2} / 2\right)-\left(\tilde{\theta}_{i, k}^{2} / 2\right)\right) \leq\left(\lambda_{i} b_{i} / 2 \gamma_{i}\right) \theta_{i, k}^{2}$.

$$
\begin{aligned}
\dot{V}_{i, k} \leq & -b_{i} 2^{(1+\alpha) / 2} \sum_{j=1}^{k} \zeta_{i, j 1}\left(\frac{1}{2} z_{i, j}^{2}+\frac{b_{i} \widetilde{\theta}_{i, j}^{2}}{2 \gamma_{i}}\right)^{(1+\alpha) / 2}-2 b_{i} \sum_{j=1}^{k} \zeta_{i, j 2}\left(\frac{1}{2} z_{i, j}^{2}+\frac{b_{i} \widetilde{\theta}_{i, j}^{2}}{2 \gamma_{i}}\right)^{(1+\beta) / 2}+\sum_{j=1}^{k} C_{j}+z_{i, k} g_{i, k} z_{i, k+1} \\
& +z_{i, k}\left(M_{i, k-1}\left(Z_{i, k}\right)-\sum_{j=1}^{k-1} \frac{\partial v_{i, k-1}}{\partial \widehat{\theta}_{i, j}} \dot{\hat{\theta}}_{i, j}\right) .
\end{aligned}
$$

From Lemmas 1, 4, and 5 and (57), we have 


$$
\begin{aligned}
-z_{i, k} \sum_{j=1}^{k-1} \frac{\partial v_{i, k-1}}{\partial \widehat{\theta}_{i, j}} \dot{\widehat{\theta}}_{i, j} & \leq-z_{i, k} \sum_{j=1}^{k-1} \frac{\partial v_{i, k-1}}{\partial \widehat{\theta}_{i, j}}\left(\frac{\gamma_{i}}{2 \eta_{i, j}^{2}} S_{i, j}^{T}\left(Z_{i, j}\right) S_{i, j}\left(Z_{i, j}\right) z_{i, j}^{2}-\lambda_{i} \widehat{\theta}_{i, j}\right) \\
& \leq \sum_{j=1}^{k-1} \frac{z_{i, k}^{2}\left(\partial v_{i, k-1} / \partial \widehat{\theta}_{i, j}\right)^{2}\left(\left(\gamma_{i} / 2 \eta_{i, j}^{2}\right) S_{i, j}^{T}\left(Z_{i, j}\right) S_{i, j}\left(Z_{i, j}\right) z_{i, j}^{2}\right)^{2}}{\sqrt{z_{i, k}^{2}\left(\partial v_{i, k-1} / \partial \widehat{\theta}_{i, j}\right)^{2}\left(\left(\gamma_{i} / 2 \eta_{i, j}^{2}\right) S_{i, j}^{T}\left(Z_{i, j}\right) S_{i, j}\left(Z_{i, j}\right) z_{i, j}^{2}\right)^{2}+\kappa^{2}}}+\lambda_{i} z_{i, k} \sum_{j=1}^{k-1} \frac{\partial v_{i, k-1}}{\partial \widehat{\theta}_{i, j}} \widehat{\theta}_{i, j} \\
& \leq \sum_{j=1}^{k-1} \frac{z_{i, k}^{2}\left(\partial v_{i, k-1} / \partial \widehat{\theta}_{i, j}\right)^{2}\left(\left(\gamma_{i} / 2 \eta_{i, j}^{2}\right) S^{2} z_{i, j}^{2}\right)^{2}}{\sqrt{z_{i, k}^{2}\left(\partial v_{i, k-1} / \partial \widehat{\theta}_{i, j}\right)^{2}\left(\left(\gamma_{i} / 2 \eta_{i, j}^{2}\right) S^{2} z_{i, j}^{2}\right)^{2}+\kappa^{2}}}+\lambda_{i} z_{i, k} \sum_{j=1}^{k-1} \frac{\partial v_{i, k-1}}{\partial \widehat{\theta}_{i, j}} \widehat{\theta}_{i, j} .
\end{aligned}
$$

Therefore, $M_{i, k-1}\left(Z_{i, k}\right)$ is defined as

$$
M_{i, k-1}\left(Z_{i, k}\right)=-\sum_{j=1}^{k-1} \frac{z_{i, k}^{2}\left(\partial v_{i, k-1} / \partial \widehat{\theta}_{i, j}\right)^{2}\left(\left(\gamma_{i} / 2 \eta_{i, j}^{2}\right) S^{2} z_{i, j}^{2}\right)^{2}}{\sqrt{z_{i, k}^{2}\left(\partial v_{i, k-1} / \partial \widehat{\theta}_{i, j}\right)^{2}\left(\left(\gamma_{i} / 2 \eta_{i, j}^{2}\right) S^{2} z_{i, j}^{2}\right)^{2}+\kappa^{2}}}-\lambda_{i} z_{i, k} \sum_{j=1}^{k-1} \frac{\partial v_{i, k-1}}{\partial \widehat{\theta}_{i, j}} \widehat{\theta}_{i, j}
$$

with the result that

$$
z_{i, k}\left(M_{i, k-1}\left(Z_{i, k}\right)-\sum_{j=1}^{k-1} \frac{\partial v_{i, k-1}}{\partial \widehat{\hat{\theta}}_{i, j}} \dot{\theta}_{i, j}\right) \leq 0
$$

$$
\dot{V}_{i, k} \leq-b_{i} 2^{(1+\alpha) / 2} \sum_{j=1}^{k} \zeta_{i, j 1}\left(\frac{1}{2} z_{i, j}^{2}+\frac{b_{i} \widetilde{\theta}_{i, j}^{2}}{2 \gamma_{i}}\right)^{(1+\alpha) / 2}-2 b_{i} \sum_{j=1}^{k} \zeta_{i, j 2}\left(\frac{1}{2} z_{i, j}^{2}+\frac{b_{i} \widetilde{\theta}_{i, j}^{2}}{2 \gamma_{i}}\right)^{(1+\beta) / 2}+\sum_{j=1}^{k} C_{j}+z_{i, k} g_{i, k} z_{i, k+1} .
$$

Step $n_{i}$. From (3) and (23), we have

$$
\begin{aligned}
\dot{z}_{i, n_{i}}= & \dot{x}_{i, n_{i}}-\dot{v}_{i, n_{i}-1}=f_{i, n_{i}}\left(\bar{x}_{i, n_{i}}, x_{i, n_{i} 0}\right)+g_{i, n_{i}}\left(u_{i}-x_{i, n_{i} 0}\right) \\
& +d_{i, n_{i}}-\dot{v}_{i, n_{i}-1},
\end{aligned}
$$

\section{Construct Lyapunov function as}

$$
V_{i, n_{i}}=V_{i, n_{i}-1}+\frac{1}{2} z_{i, n_{i}}^{2}+\frac{b_{i} \widetilde{\theta}_{i, n_{i}}^{2}}{2 \gamma_{i}}
$$

The derivative of $V_{i, n_{i}}$ is

where $\dot{v}_{i, n_{i}-1}=\sum_{j=1}^{n_{i}-1}\left(\partial v_{i, n_{i}-1} / \partial x_{i, j}\right) \dot{x}_{i, j}+h_{i} \sum_{j=1}^{n_{i}}\left(\partial v_{i, n_{i}-1} /\right.$

$\left.\partial x_{0, j}\right) \dot{x}_{0, j}+\sum_{j=1}^{M} a_{i j} \sum_{q=1}^{n_{i}}\left(\left(\partial v_{i, n_{i}-1} / \partial x_{j, q}\right) \dot{x}_{j, q}\right)+\sum_{j=1}^{n_{i}-1}$

$\left(\partial v_{i, n_{i}-1} / \partial \widehat{\theta}_{i, j}\right) \hat{\hat{\theta}}_{i, j}+\backslash \backslash \sum_{j=0}^{n_{i}-1}\left(\partial v_{i, n_{i}-1} / \partial F_{b}^{(j)}\right) F_{b}^{(j+1)}$.

$$
\begin{aligned}
& \dot{V}_{i, n_{i}} \leq-b_{i} 2^{(1+\alpha) / 2} \sum_{j=1}^{n_{i}-1} \zeta_{i, j 1}\left(\frac{1}{2} z_{i, j}^{2}+\frac{b_{i} \widetilde{\theta}_{i, j}^{2}}{2 \gamma_{i}}\right)^{(1+\alpha) / 2}-2 b_{i} \sum_{j=1}^{n_{i}-1} \zeta_{i, j 2}\left(\frac{1}{2} z_{i, j}^{2}+\frac{b_{i} \widetilde{\theta}_{i, j}^{2}}{2 \gamma_{i}}\right)^{(1+\beta) / 2} \\
& +z_{i, n_{i}} \widehat{f}_{i, n_{i}}\left(Z_{i, n_{i}}\right)+z_{i, n_{i}} g_{i, n_{i}} u_{i}-z_{i, n_{i}} g_{i, n_{i}} x_{i, n_{i} 0}-z_{i, n_{i}} \sum_{j=1}^{n_{i}-1} \frac{\partial v_{i, n_{i}-1}}{\partial \widehat{\hat{\theta}}_{i, j}} \hat{\widehat{\theta}}_{i, j}-\frac{b_{i} \widetilde{\theta}_{i, n_{i}} \dot{\hat{\theta}}_{i, n_{i}}}{\gamma_{i}}+z_{i, n_{i}} M_{i, n_{i}-1}\left(Z_{i, n_{i}}\right)+\sum_{j=1}^{n_{i}-1} C_{i, j},
\end{aligned}
$$

where 


$$
\begin{aligned}
\hat{f}_{i, n_{i}}\left(Z_{i, n_{i}}\right)= & f_{i, n_{i}}\left(\bar{x}_{i, n_{i}}, x_{i, n_{i} 0}\right)+d_{i, n_{i}}+z_{i, n_{i}-1} g_{i, n_{i}-1}-\sum_{j=1}^{n_{i}-1} \frac{\partial v_{i, n_{i}-1}}{\partial x_{i, j}} \dot{x}_{i, j}-\sum_{j=1}^{M} a_{i j} \sum_{q=1}^{n_{i}}\left(\frac{\partial v_{i, n_{i}-1}}{\partial x_{j, q}} \dot{x}_{j, q}\right) \\
& -h_{i} \sum_{j=1}^{n_{i}} \frac{\partial v_{i, n_{i}-1}}{\partial x_{0, j}} \dot{x}_{0, j}-\sum_{j=0}^{n_{i}-1} \frac{\partial v_{i, n_{i}-1}}{\partial F_{b}^{(j)}} F_{b}^{(j+1)}-M_{i, n_{i}-1}\left(Z_{i, n_{i}}\right) .
\end{aligned}
$$

The actual controller is defined as

$$
\begin{aligned}
u_{i}= & -\zeta_{i, n_{i} 1} S_{z_{i, n_{i}} 1}-\zeta_{i, n_{i}} S_{z_{i, n_{i}}, 2}-\frac{\hat{\theta}_{i, n_{i}}}{2 \eta_{i, n_{i}}^{2}} S_{i, n_{i}}^{T}\left(Z_{i, n_{i}}\right) S_{i, n_{i}}\left(Z_{i, n_{i}}\right) z_{i, n_{i}} \\
& -\zeta_{i, n_{i} 3} z_{i, n_{i}}+x_{i, n_{i} 0},
\end{aligned}
$$

where $\zeta_{i, n_{i} 1}, \quad \zeta_{i, n_{i} 2}, \quad \zeta_{i, n_{i} 3}$, and $\eta_{i, n_{i}}$ are positive design parameters.

The adaptive law $\widehat{\theta}_{i, n_{i}}$ is defined as

$$
\dot{\hat{\theta}}_{i, n_{i}}=\frac{\gamma_{i}}{2 \eta_{i, n_{i}}^{2}} S_{i, n_{i}}^{T}\left(Z_{i, n_{i}}\right) S_{i, n_{i}}\left(Z_{i, n_{i}}\right) z_{i, n_{i}}^{2}-\lambda_{i} \widehat{\theta}_{i, n_{i}} \text {. }
$$

$$
\begin{aligned}
\dot{V}_{i, n_{i}} \leq & -b_{i} 2^{(1+\alpha) / 2} \sum_{j=1}^{n_{i}-1} \zeta_{i, j 1}\left(\frac{1}{2} z_{i, j}^{2}+\frac{b_{i} \widetilde{\theta}_{i, j}^{2}}{2 \gamma_{i}}\right)^{(1+\alpha) / 2}-2 b_{i} \sum_{j=1}^{n_{i}-1} \zeta_{i, j 2}\left(\frac{1}{2} z_{i, j}^{2}+\frac{b_{i} \widetilde{\theta}_{i, j}^{2}}{2 \gamma_{i}}\right)^{(1+\beta) / 2}-b_{i} \zeta_{i, n_{i} 1} z_{i, n_{i}} S_{z_{i, n_{i}}, 1}-b_{i} \zeta_{i, n_{i} 2} z_{i, n_{i}} S_{z_{i, n_{i}}, 2} \\
& -b_{i} \zeta_{i, n_{i} 1} 2^{(1+\alpha) / 2}\left(\frac{b_{i} \widetilde{\theta}_{i, n_{i}}^{2}}{2 \gamma_{i}}\right)^{(1+\alpha) / 2}-b_{i} \zeta_{i, n_{i} 2} 2^{(1+\beta) / 2}\left(\frac{b_{i} \widetilde{\theta}_{i, n_{i}}^{2}}{2 \gamma_{i}}\right)^{(1+\beta) / 2}+z_{i, n_{i}}\left(M_{i, n_{i}-1}\left(Z_{i, n_{i}}\right)-\sum_{j=1}^{n_{i}-1} \frac{\partial v_{i, n_{i}-1}}{\partial \widehat{\hat{\theta}}_{i, j}}\right)_{i, j}+\sum_{j=1}^{n_{i}} C_{i, j},
\end{aligned}
$$

where $C_{i, n_{i}}=\sigma_{i, n_{i}}+\beta_{i, n_{i}}+\left(\lambda_{i} b_{i} / 2 \gamma_{i}\right) \theta_{i, n_{i}}^{2}, \beta_{l, n_{i}}=b_{i} \zeta_{i, n_{i} 1} 2^{(1+\alpha) / 2}$ $\left(b_{i} Q_{l, n_{i}}^{2} / 2 \gamma_{i}\right)^{(1+\alpha) / 2}+b_{i} \zeta_{l, n_{i} 2^{2}} 2^{(1+\beta) / 2}\left(b_{i} Q_{i, n_{i}}^{2} / 2 \gamma_{i}\right)^{(1+\beta) / 2}, \quad \sigma_{i, n_{i}}=$ According to (27), (28), (32), and (33) and when $(1 / 2) \eta_{i, n_{i}}^{2}+\left(\varepsilon_{i, n_{i}}^{2} / 4 b_{i} \zeta_{i, n_{i} 3}\right)$, and $\left(\lambda_{i} b_{i} \widetilde{\theta}_{i, n_{i}} \widehat{\theta}_{i, n_{i}} / \gamma_{i}\right) \leq\left(\lambda_{i} b_{i} / \gamma_{i}\right)$ $\left(\left(\theta_{i, n_{i}}^{2} / 2\right)-\left(\tilde{\theta}_{i, n_{i}}^{2} / 2\right)\right) \leq\left(\lambda_{i} b_{i} / 2 \gamma_{i}\right) \theta_{i, n_{i}}^{2}$.

$$
\begin{aligned}
\dot{V}_{i, n_{i}} \leq & \left.-b_{i} 2^{(1+\alpha) / 2} \sum_{j=1}^{n_{i}} \zeta_{i, j 1}\left(\frac{1}{2} z_{i, j}^{2}+\frac{b_{i} \widetilde{\theta}_{i, j}^{2}}{2 \gamma_{i}}\right)^{(1+\alpha) / 2}-2 b_{i} \sum_{j=1}^{n_{i}} \zeta_{i, j 2}\left(\frac{1}{2} z_{i, j}^{2}+\frac{b_{i} \widetilde{\theta}_{i, j}^{2}}{2 \gamma_{i}}\right)^{(1+\beta) / 2}+z_{i, n_{i}}\left(M_{i, n_{i}-1}\left(Z_{i, n_{i}}\right)-\sum_{j=1}^{n_{i}-1} \frac{\partial v_{i, n_{i}-1}}{\partial \widehat{\hat{\theta}}_{i, j}}\right)_{i, j}\right) \\
& +\sum_{j=1}^{n_{i}} C_{i, j} .
\end{aligned}
$$

Same to the process of $(60)$, the $M_{i, n_{i}-1}\left(Z_{i, n_{i}}\right)$ can be defined as

$$
M_{i, n_{i}-1}\left(Z_{i, n_{i}}\right)=-\sum_{j=1}^{n_{i}-1} \frac{z_{i, n_{i}}^{2}\left(\partial v_{i, n_{i}-1} / \partial \widehat{\theta}_{i, j}\right)^{2}\left(\left(\gamma_{i} / 2 \eta_{i, j}^{2}\right) S^{2} z_{i, j}^{2}\right)^{2}}{\sqrt{z_{i, k}^{2}\left(\partial v_{i, n_{i}-1} / \partial \hat{\theta}_{i, j}\right)^{2}\left(\left(\gamma_{i} / 2 \eta_{i, j}^{2}\right) S^{2} z_{i, j}^{2}\right)^{2}+\kappa^{2}}}-\lambda_{i} z_{i, n_{i}} \sum_{j=1}^{n_{i}-1} \frac{\partial v_{i, n_{i}-1}}{\partial \widehat{\theta}_{i, j}} \widehat{\theta}_{i, j}
$$

with the result that 


$$
z_{i, n_{i}}\left(M_{i, n_{i}-1}\left(Z_{i, n_{i}}\right)-\sum_{j=1}^{n_{i}-1} \frac{\partial v_{i, n_{i}-1}}{\partial \widehat{\hat{\theta}}_{i, j}}{ }_{i, j}\right) \leq 0
$$

Substituting (73) into (71) yields

$$
\dot{V}_{i, n_{i}} \leq-b_{i} 2^{(1+\alpha) / 2} \sum_{j=1}^{n_{i}} \zeta_{i, j 1}\left(\frac{1}{2} z_{i, j}^{2}+\frac{b_{i} \widetilde{\theta}_{i, j}^{2}}{2 \gamma_{i}}\right)^{(1+\alpha) / 2}-2 b_{i} \sum_{j=1}^{n_{i}} c_{i, j 2}\left(\frac{1}{2} z_{i, j}^{2}+\frac{b_{i} \widetilde{\theta}_{i, j}^{2}}{2 \gamma_{i}}\right)^{(1+\beta) / 2}+\tau_{i}
$$

where $\tau_{i}=\sum_{j=1}^{n_{i}} C_{i, j}$.

Setting $\phi_{i, 1}=\min b_{i} 2^{(1+\alpha) / 2}\left\{\zeta_{i, 11}, \zeta_{i, 21}, \ldots, \zeta_{i, n_{1}, 1}\right\}$ and $\phi_{i, 2}=\min 2 b_{i}\left\{\zeta_{i, 12}, \zeta_{i, 22}, \ldots, \zeta_{i, n_{i}}\right\}$ and from Lemma 3, we have

$$
\begin{aligned}
&-b_{i} 2^{(1+\alpha) / 2} \sum_{j=1}^{n_{i}} \zeta_{i, j 1}\left(\frac{1}{2} z_{i, j}^{2}+\frac{b_{i} \widetilde{\theta}_{i, j}^{2}}{2 \gamma_{i}}\right)^{(1+\alpha) / 2} \leq-\phi_{i, 1}\left(\sum_{j=1}^{n_{i}}\left(\frac{1}{2} z_{i, j}^{2}+\frac{b_{i} \widetilde{\theta}_{i, j}^{2}}{2 \gamma_{i}}\right)\right)^{(1+\alpha) / 2}, \\
&-2 b_{i} \sum_{j=1}^{n_{i}} \zeta_{i, j 2}\left(\frac{1}{2} z_{i, j}^{2}+\frac{b_{i} \widetilde{\theta}_{i, j}^{2}}{2 \gamma_{i}}\right)^{(1+\beta) / 2} \leq-n_{i}^{(1-\beta) / 2} \phi_{i, 2}\left(\sum_{j=1}^{n_{i}}\left(\frac{1}{2} z_{i, j}^{2}+\frac{b_{i} \widetilde{\theta}_{i, j}^{2}}{2 \gamma_{i}}\right)\right)^{(1+\beta) / 2} .
\end{aligned}
$$

Then, (74) can be rewritten as

$$
\dot{V}_{i, n_{i}} \leq-\phi_{i, 1} V_{i, n_{i}}^{(1+\alpha) / 2}-n_{i}^{(1-\beta) / 2} \phi_{i, 2} V_{i, n_{i}}^{(1+\beta) / 2}+\tau_{i} .
$$

Up to this point, the design of fixed-time controller is finished.

\section{Stability Analysis}

Theorem 1. Consider pure-feedback nonlinear multiagent systems (1) satisfying Assumptions 1-3. If given initial values $-\sigma_{2} F_{b}(0)<e_{i}(0)<\sigma_{1} F_{b}(0)$, then by utilizing virtual controllers (31), (43), and (52), actual controller (68) and adaptive laws (37), (45), (57), and (69) can ensure that the local consensus errors converge to a small neighborhood of the origin within a fixed time. Besides, the local tracking errors can meet the prescribed performance range, i.e., $-\sigma_{2} F_{b}(t)<e_{i}(t)<\sigma_{1} F_{b}(t)$.

Proof. Choose the total Lyapunov function $V$ as follows:

$$
V=\sum_{i=1}^{M} V_{i, n_{i}}
$$

According to the process above and (76), the derivative of $V$ can be written as

$$
\dot{V} \leq-\sum_{i=1}^{M} \phi_{i, 1} V_{i, n_{i}}^{(1+\alpha) / 2}-\sum_{i=1}^{M} n_{i}^{(1-\beta) / 2} \phi_{i, 2} V_{i, n_{i}}^{(1+\beta) / 2}+\sum_{i=1}^{M} \tau_{i} .
$$

Setting $\mu_{1}=\min \left\{\phi_{1,1}, \phi_{2,1}, \ldots, \phi_{M, 1}\right\}, \tau=\sum_{i=1}^{M} \tau_{i}$, and $\mu_{2}=\min \left\{n_{1}^{(1-\beta) / 2} M^{(1-\beta) / 2} \phi_{1,2}, \ldots, n_{M}^{(1-\beta) / 2} M^{(1-\beta) / 2} \phi_{M, 2}\right\}$ and from Lemma 3, we have

$$
-\sum_{i=1}^{M} \phi_{i, 1} V_{i, n_{i}}^{(1+\alpha) / 2} \leq-\mu_{1}\left(\sum_{i=1}^{M} V_{i, n_{i}}\right)^{(1+\alpha) / 2}
$$

$$
-\sum_{i=1}^{M} n_{i}^{(1-\beta) / 2} \phi_{i, 2} V_{i, n_{i}}^{(1+\beta) / 2} \leq-\mu_{2}\left(\sum_{i=1}^{M} V_{i, n_{i}}\right)^{(1+\beta) / 2},
$$

where $\mu_{1}>0, \mu_{2}>0, \alpha \in(0,1]$, and $\beta \in(1, \infty)$.

Furthermore, substituting (79) and (80) into (78), we have

$$
\dot{V} \leq-\mu_{1} V^{(1+\alpha) / 2}-\mu_{2} V^{(1+\beta) / 2}+\tau .
$$

According to Lemma 2, choose the appropriate parameters to make (81) satisfy the following conditions.

Case 1. If $V>\left(\tau /\left((1-\varpi) \mu_{1}\right)\right)^{2 /(1+\alpha)}, \varpi \in(0,1)$, then (81) can be written as

$$
\dot{V} \leq-\bowtie \mu_{1} V^{(1+\alpha) / 2}-\mu_{2} V^{(1+\beta) / 2} .
$$

The solution of system (1) converges on the compact set,

$$
x \in\left\{V(x) \leq\left(\frac{\tau}{(1-\varpi) \mu_{1}}\right)^{2 /(1+\alpha)}\right\} .
$$

The fixed convergence time is 


$$
T \leq T_{\max }:=\frac{2}{\varpi \mu_{1}(1-\alpha)}+\frac{2}{\mu_{2}(\beta-1)} .
$$

Case 2. If $V>\left(\tau /\left((1-\Phi) \mu_{2}\right)\right)^{2 /(1+\beta)}, \omega \in(0,1)$, then $(81)$ can be written as

$$
\dot{V} \leq-\mu_{1} V^{(1+\alpha) / 2}-\Phi \mu_{2} V^{(1+\beta) / 2} .
$$

The solution of system (1) converges on the compact set,

$$
x \in\left\{V(x) \leq\left(\frac{\tau}{(1-\Phi) \mu_{2}}\right)^{2 /(1+\beta)}\right\} \text {. }
$$

The fixed convergence time is

$$
T \leq T_{\max }:=\frac{2}{\mu_{1}(1-\alpha)}+\frac{2}{\varpi \mu_{2}(\beta-1)} .
$$

Combining Cases 1 and 2, the solution of system (1) converges on

$$
x \in\left\{V(x) \leq \min \left\{\left(\frac{\tau}{(1-\Phi) \mu_{1}}\right)^{2 /(1+\alpha)},\left(\frac{\tau}{(1-\Phi) \mu_{2}}\right)^{2 /(1+\beta)}\right\}\right\} .
$$

The fixed convergence time is

$$
T_{s} \leq T_{\max }:=\frac{2}{\varpi \mu_{1}(1-\alpha)}+\frac{2}{\varpi \mu_{2}(\beta-1)} .
$$

It can be seen from (82) and (85) that $V$ is bounded, with the result that $z_{i, j}$ and $\tilde{\theta}_{i, j}$ are bounded, where $i=1, \ldots, M$, $j=1, \ldots, n_{i}$, as $\widehat{\theta}_{i, j}=\theta_{i, j}-\widetilde{\theta}_{i, j}$ is also bounded. Because $e_{i}=$ $\sum_{j=1}^{M} a_{i j}\left(y_{i}-y_{j}\right)+h_{i}\left(y_{i}-y_{0}\right)$ and $e_{i}$ and $y_{0}$ are bounded, $y_{i}$ are bounded. Because $z_{i, 2}=x_{i, 2}-v_{i, 1}, x_{i, 2}$ are bounded. Similarly, $v_{i, k-1}$ and $x_{i, k}, k=1, \ldots, n_{i}$ are bounded. Therefore, all signals in the overall closed-loop system (1) are bounded.

Remark 5. In this paper, the adaptive fixed-time tracking consensus problem has been solved for pure-feedback nonlinear MASs. Compared with existing results [16-18, 24-27] on high-order MASs with nonlinear strictfeedback, our studied system is more general. Furthermore, the introduction of tracking error constraint extends the application scope of existing fixed-time control methods.

Remark 6. In fact, when the structure of the system is too complex, the design of the controller is very difficult. In this paper, the RBF neural network is used to approximate the uncertain nonlinear function, so the precise system dynamics structure is not needed when designing the controller.

\section{Simulation Results}

In this section, two simulation examples are employed to verify the controller designed as described in the above paragraphs.

5.1. Example 1: Numerical Example. Consider the secondorder nonlinear MASs consisting of four followers and one leader, and the dynamic of each agent $N_{i}$ is given as the following pure-feedback form:

$$
\left\{\begin{array}{l}
\dot{x}_{i, 1}=\frac{1-e^{-x_{i, 1}}}{1+e^{-x_{i, 2}}}+x_{i, 2}^{3}+x_{i, 2} e^{-1-x_{i, 1}^{2}}+d_{i, 1}\left(\bar{x}_{i, 1}, t\right) \\
\dot{x}_{i, 2}=x_{i, 1}^{2} x_{i, 2}+\left(2 u_{i}+u_{i}^{3}\right)\left(x_{i, 1}^{2}+x_{i, 2}^{2}\right)+d_{i, 2}\left(\bar{x}_{2}, t\right) \\
y_{i}=x_{i, 1},
\end{array}\right.
$$

where $x_{i, 1}$ and $x_{i, 2}$, with $i=1, \ldots, 4$, are the state variables, $u_{i}$ is the system input, and $y_{i}$ is the system output. $d_{i, 1}\left(\bar{x}_{i, 1}, t\right)=$ $0.7 x_{i, 1}^{2} \cos (1.5 t)$ and $d_{i, 2}\left(\bar{x}_{i, 2}, t\right)=0.5\left(x_{i, 1}^{2}+x_{i, 2}^{2}\right) \sin ^{3}(t)$ are the external disturbance terms.

The directed network topology for the simulation is shown in Figure 1. We choose $a_{i j}=1$ on $j \in N_{i}, a_{i j}=0$; otherwise, $h_{1}=h_{2}=h_{3}=0$, and $h_{4}=1$. The leader's output $y_{0}(t)$ is $0.5 \sin (1.5 t)+\cos (0.5 t)$ and the initial states and adaptive laws of the four followers are set to $x_{1}(0)=[0.5,0.5]^{T}, x_{2}(0)=[0.1,0.1]^{T}, x_{3}(0)=[-0.3,-0.3]$ $T$, and $x_{4}(0)=[0.3,0.3]^{T}$, where $x_{i}(0)=\left[x_{i, 1}(0), x_{i, 2}(0)\right]^{T}$ with $i=1, \ldots, 4$, and $\widehat{\theta}_{i}=\left[\hat{\theta}_{i, 1}, \widehat{\theta}_{i, 2}\right]=[0,0]^{T}$ with $i=1, \ldots, 4$. To display the tracking errors between the leader and the followers, we use $e_{i}=\left|y_{i}(t)-y_{0}(t)\right|$, where $i=1, \ldots, 4$, and the design parameters are chosen as follows: $\alpha=1 / 3, \quad \beta=2, \quad \zeta_{i, 11}=\zeta_{i, 12}=\zeta_{i, 21}=\zeta_{i, 22}=0.2, \quad \zeta_{i, 13}=\zeta_{i, 23}$ $=10, \quad b_{i}=5, \quad \gamma_{i}=5, \quad \eta_{i, 1}=\eta_{i, 2}=0.25, \quad \varepsilon_{10}=\varepsilon_{20}=0.001$, $\lambda_{i}=0.04, x_{i, 10}=0.2$, and $x_{i, 20}=0.5$, where $i=1, \ldots, 4$. It is worth noting that $\zeta_{i, 13}=\zeta_{i, 23}$ should be at least ten times that of $\zeta_{i, 11}=\zeta_{i, 12}=\zeta_{i, 21}=\zeta_{i, 22}$ and $b_{i}$ setting of 5 is much better than setting it to 2 . The performance boundary function's design parameters $\xi_{0}, a, \xi_{\infty}, \sigma_{1}$, and $\sigma_{2}$ are chosen as $0.9,1$, $0.17,2$, and 2 , respectively.

Then, the virtual/actual control signals and the adaptive laws are, respectively, designed as follows: 


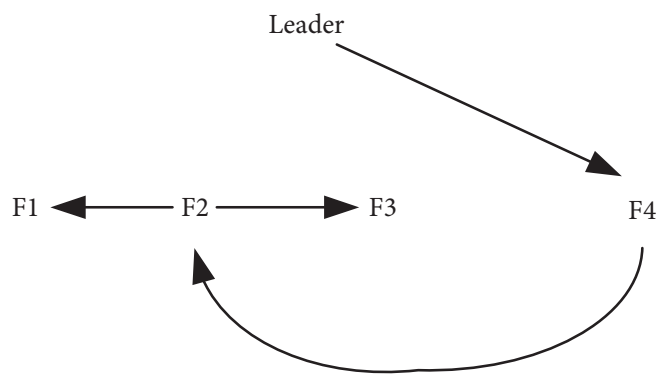

Figure 1: Network topology for a group of one leader and four followers F1 to F4.

$$
\begin{aligned}
& v_{i, 1}=\frac{1}{\left(d_{i}+h_{i}\right) F_{b} \Phi_{F_{i}}}-\left(\frac{\hat{\theta}_{i, 1}}{2 \eta_{i, 1}^{2}} S_{i, 1}^{T}\left(Z_{i, 1}\right) S_{i, 1}\left(Z_{i, 1}\right) z_{i, 1}-\zeta_{i, 11} S_{z_{i, 1}, 1}-\zeta_{i, 12} S_{z_{i, 1}, 2}-\zeta_{i, 13} z_{i, 1}+x_{i, 10}\right), \\
& u_{i}=-\zeta_{i, 21} S_{z_{i, 2}, 1}-\zeta_{i, 22} S_{z_{i, 2}, 2}-\frac{\widehat{\theta}_{i, 2}}{2 \eta_{i, 2}^{2}} S_{i, 2}^{T}\left(Z_{i, 2}\right) S_{i, 2}\left(Z_{i, 2}\right) z_{i, 2}-\zeta_{i, 23} z_{i, 2}+x_{i, 20}, \\
& \dot{\hat{\theta}}_{i, 1}=\frac{\gamma_{i}}{2 \eta_{i, 1}^{2}} S_{i, 1}^{T}\left(Z_{i, 1}\right) S_{i, 1}\left(Z_{i, 1}\right) z_{i, 1}^{2}-\lambda_{i} \widehat{\theta}_{i, 1}, \\
& \dot{\hat{\theta}}_{i, 2}=\frac{\gamma_{i}}{2 \eta_{i, 2}^{2}} S_{i, 2}^{T}\left(Z_{i, 2}\right) S_{i, 2}\left(Z_{i, 2}\right) z_{i, 2}^{2}-\lambda_{i} \widehat{\theta}_{i, 2},
\end{aligned}
$$

where $i=1, \ldots, 4$.

In this simulation example, we set the width of the RBF neural network as four. $W_{i, 1} S_{i, 1}\left(Z_{i, 1}\right)$ and $W_{i, 2} S_{i, 2}\left(Z_{i, 2}\right)$ contain seven and five nodes, respectively. The center of the Gaussian function is set as

$$
\begin{aligned}
\xi_{i, 1} & =\left[\begin{array}{rrrrrrr}
-2 & -1.5 & -1 & 0 & 1 & 1.5 & 2 \\
-2 & -1.5 & -1 & 0 & 1 & 1.5 & 2 \\
-2 & -1.5 & -1 & 0 & 1 & 1.5 & 2 \\
-2 & -1.5 & -1 & 0 & 1 & 1.5 & 2 \\
-2 & -1.5 & -1 & 0 & 1 & 1.5 & 2 \\
-2 & -1.5 & -1 & 0 & 1 & 1.5 & 2
\end{array}\right], \\
\xi_{i, 2} & =\left[\begin{array}{rrrrr}
-2 & -1 & 0 & 1 & 2 \\
-2 & -1 & 0 & 1 & 2 \\
-2 & -1 & 0 & 1 & 2 \\
-3 & -2 & 0 & 2 & 3 \\
-3 & -2 & 0 & 2 & 3 \\
-3 & -2 & 0 & 2 & 3 \\
-3 & -2 & 0 & 2 & 3 \\
-3 & -2 & 0 & 2 & 3 \\
-3 & -2 & 0 & 2 & 3 \\
-3 & -2 & 0 & 2 & 3
\end{array}\right] .
\end{aligned}
$$

In Figure 2, the tracking curves of the output signals $y_{i}$ are presented. It can be seen that, by utilizing the proposed fixed-time control strategy, the followers can fast track the leader. In Figure 3, the errors between the followers and the leader are shown. It can be seen that all local tracking errors are within the prescribed boundaries. In Figure 4, the responses of adaptive laws in MASs are given, which shows that adaptive signals $\widehat{\theta}_{i, j}(i=1, \ldots, 4, j=1,2)$ are all bounded for $t \geq 0$. In Figure 5 , the responses of control inputs in MASs are given, which shows that control input signals $u_{i}(i=1, \ldots, 4)$ are all bounded for $t \geq 0$. All the above simulation results illustrate the validity of the proposed method.

5.2. Example 2: Physical Example. Consider the following multiple electromechanical systems consisting of two followers and one leader. The dynamic of each electromechanical system is described as

$$
\begin{aligned}
& M \ddot{q}+B \dot{q}+N \sin q+\Delta_{1}(\dot{q}, q, I)=I \\
& \dot{L} \dot{I}=V_{e}-R I-K_{B} \dot{q}+\Delta_{1}(\dot{q}, q, I),
\end{aligned}
$$

where $q$ is the angular motor position, $V_{e}$ is the input control voltage, and $I$ is the motor armature, $M=\left(J / K_{\tau}\right)+$ $\left(m L_{0}^{2} / 3 K_{\tau}\right)+\left(M_{0} L_{0}^{2} / K_{\tau}\right)+\left(2 M_{0} R_{0}^{2} / 5 K_{\tau}\right), \quad B=\left(B_{0} / K_{\tau}\right)$, and $N=\left(m L_{0} g / 2 K_{\tau}\right)+\left(M_{0} L_{0} g / K_{\tau}\right), \Delta_{1}(\dot{q}, q)$, and $\Delta_{2}(\dot{q}, q)$ denote the model errors. $J$ denotes the rotor inertia, $K_{\tau}$ is the coefficient to characterize the electromechanical conversion of armature current to torque, $L_{0}$ is the link length, $m$ means the link mass, $M_{0}$ stands for the load mass, $B_{0}$ is the coefficient of viscous friction at the joint, $R_{0}$ is the radius of the load, $g$ is the gravity coefficient, $R$ is the armature resistance, $L$ is the armature inductance, and $K_{B}$ is the back-emf coefficient. 


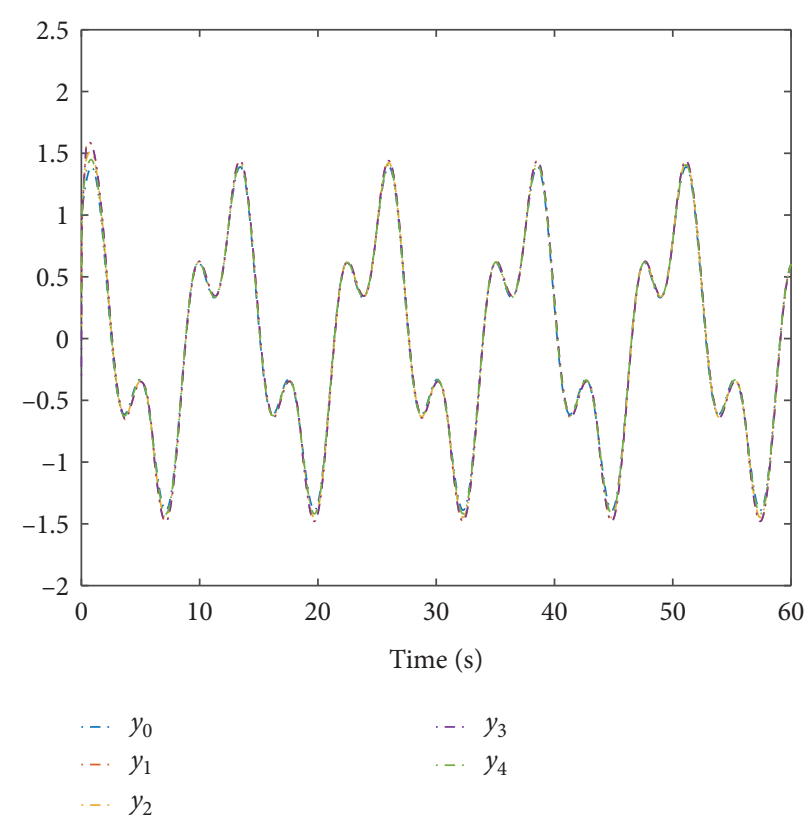

FIgURE 2: Response curves of the output signals $y_{i}$ and $y_{0}$.

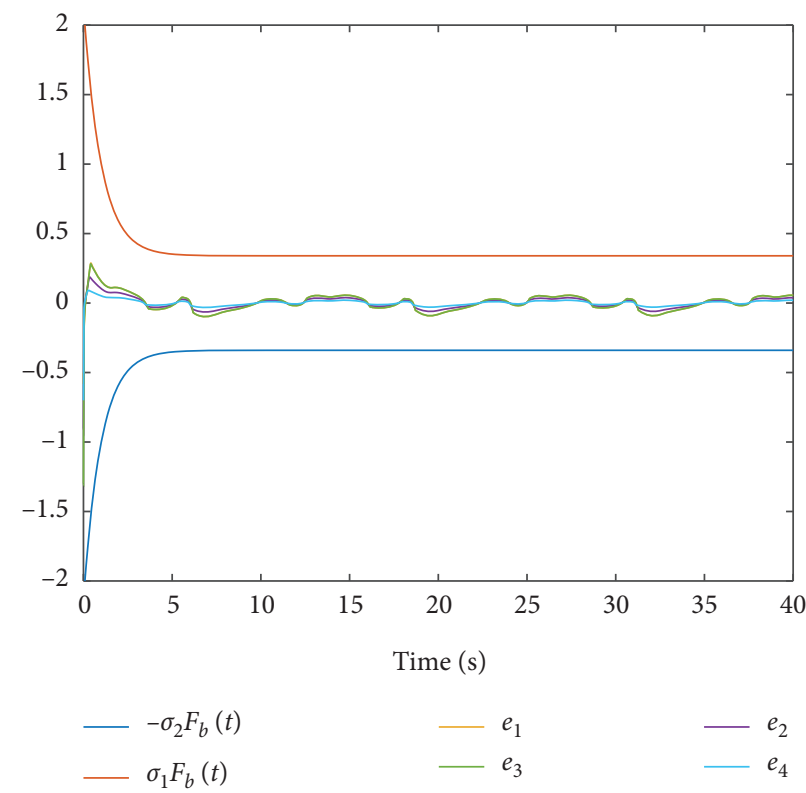

FIgURE 3: Response curves of the tracking consensus error $e_{i}$.

Let $x_{i, 1}=q, x_{i, 2}=\dot{q}, x_{i, 3}=I, y_{i}=x_{i, 1}$, and $u_{i}=V_{e}$. Then, the electromechanical system can be rewritten as

$$
\left\{\begin{array}{l}
\dot{x}_{i, 1}=x_{i, 2} \\
\dot{x}_{i, 2}=e_{21} x_{i, 2} x_{i, 3}^{2}+e_{22} \sin x_{i, 1}+e_{23} x_{i, 2}-e_{23} x_{i, 2}^{2} x_{i, 3}^{3}, \\
\dot{x}_{i, 3}=e_{31} u_{i}+e_{32} x_{i, 2}+e_{33} x_{i, 3}^{2}-e_{33} x_{i, 2}^{2} \sin x_{i, 3}, \\
y_{i}=x_{i, 1}
\end{array}\right.
$$

where $e_{21}=1 / M, \quad e_{22}=-N / M, \quad e_{23}=-B / M, \quad e_{31}=1 / L$, $e_{32}=-K_{B} / L$, and $e_{33}=-R / L$.

Assume that there are three followers and one leader in MASs, and the communications are $h_{1}=1, h_{2}=h_{3}=0$, $a_{2,1}=a_{3,1}=1$, and $a_{1,2}=a_{1,3}=a_{2,3}=a_{3,2}=0$. The parameters are chosen as follows: $M=0.0642, N=1.1408$, $B=0.0181, L=0.025, K_{B}=0.9$, and $R=5.0$. The leader's output $y_{0}(t)$ is $\sin (0.5 t)+0.5 \sin (t)$. The predefined performance function' parameters $\xi_{0}, a, \xi_{\infty}, \sigma_{1}$, and $\sigma_{2}$ are chosen as $0.9,7,0.17,1$, and 1 , respectively. The purpose of 

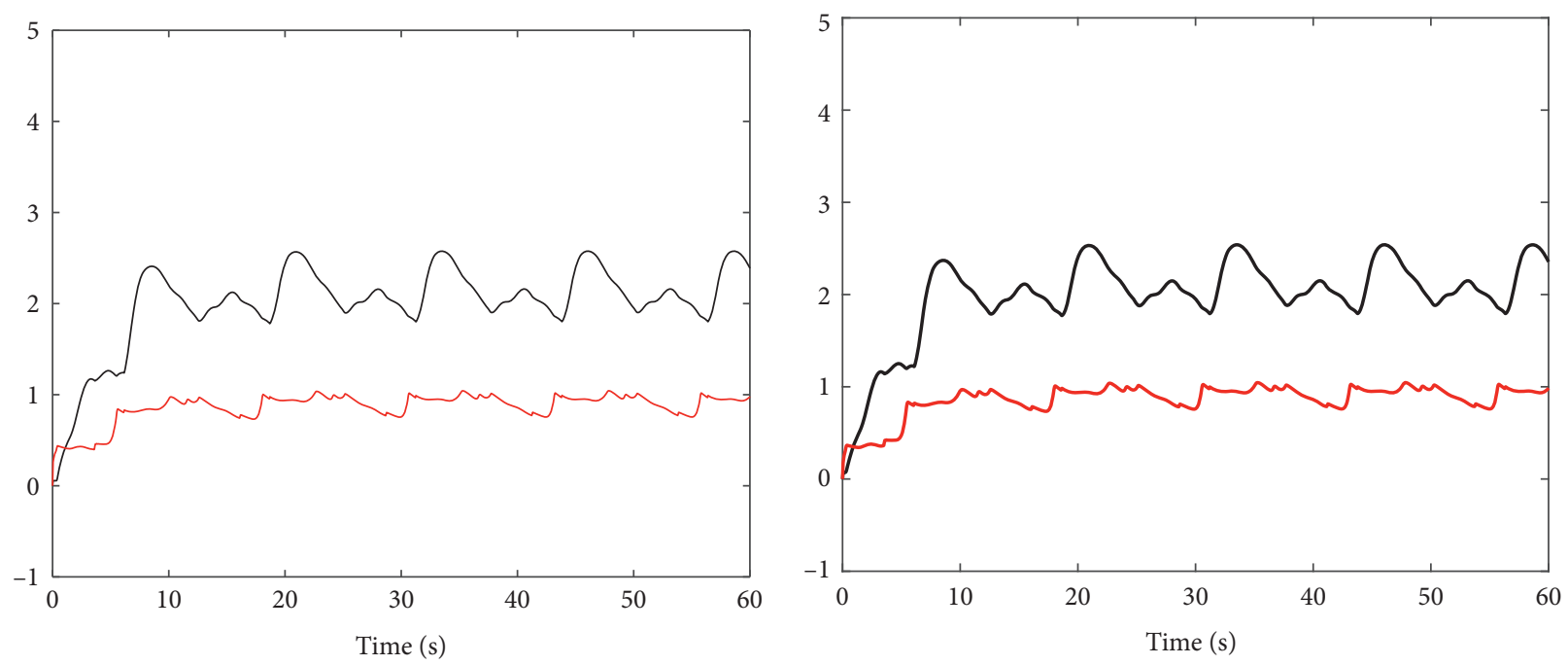

$$
\begin{aligned}
& \hat{\theta}_{1,1} \\
& -\hat{\theta}_{1,2}
\end{aligned}
$$

(a)

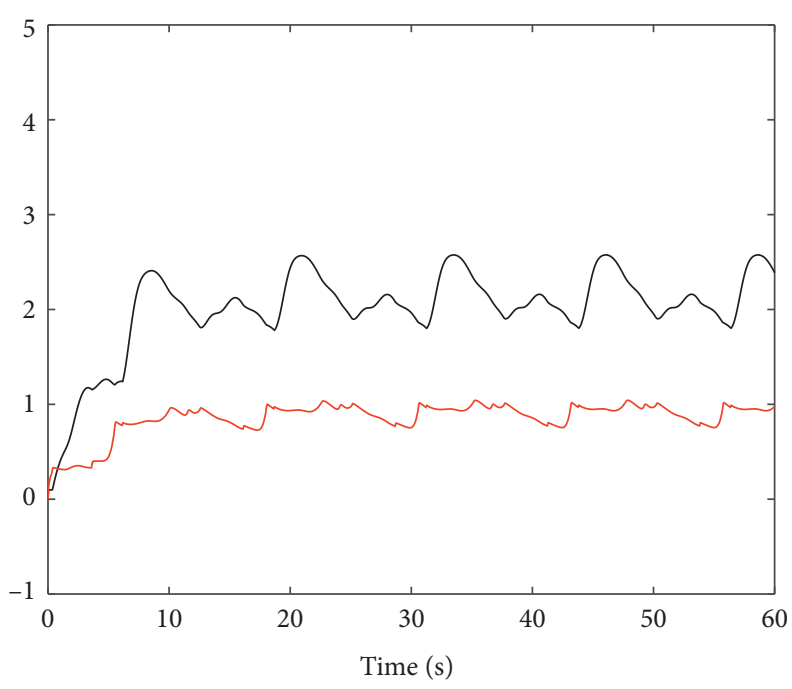

$-\hat{\theta}_{3,1}$
$-\hat{\theta}_{3,2}$

$$
\begin{aligned}
& -\hat{\theta}_{2,1} \\
& -\hat{\theta}_{2,2}
\end{aligned}
$$

(b)

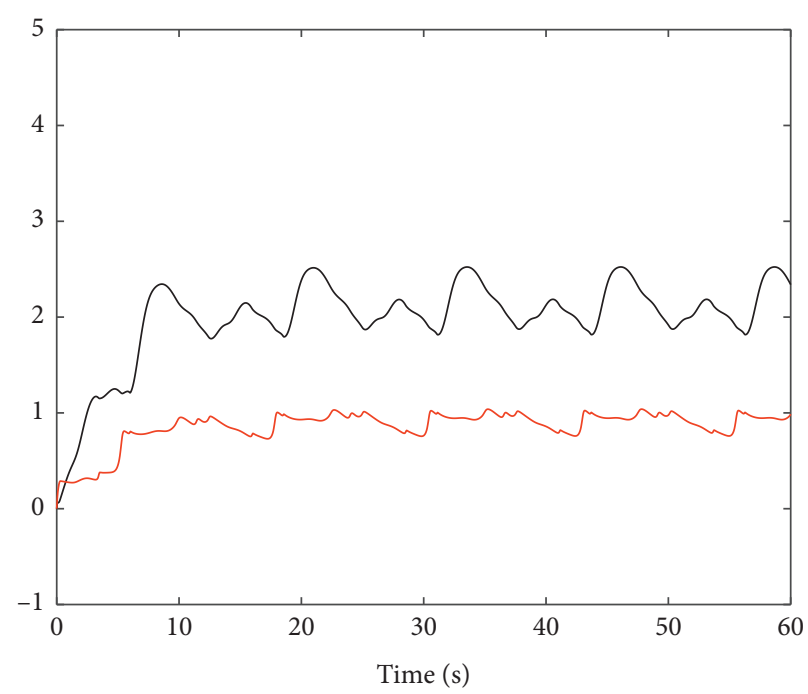

$$
\begin{aligned}
& -\hat{\theta}_{4,1} \\
& -\hat{\theta}_{4,2}
\end{aligned}
$$

(d)

FIgUre 4: Adaptive laws in MASs. 

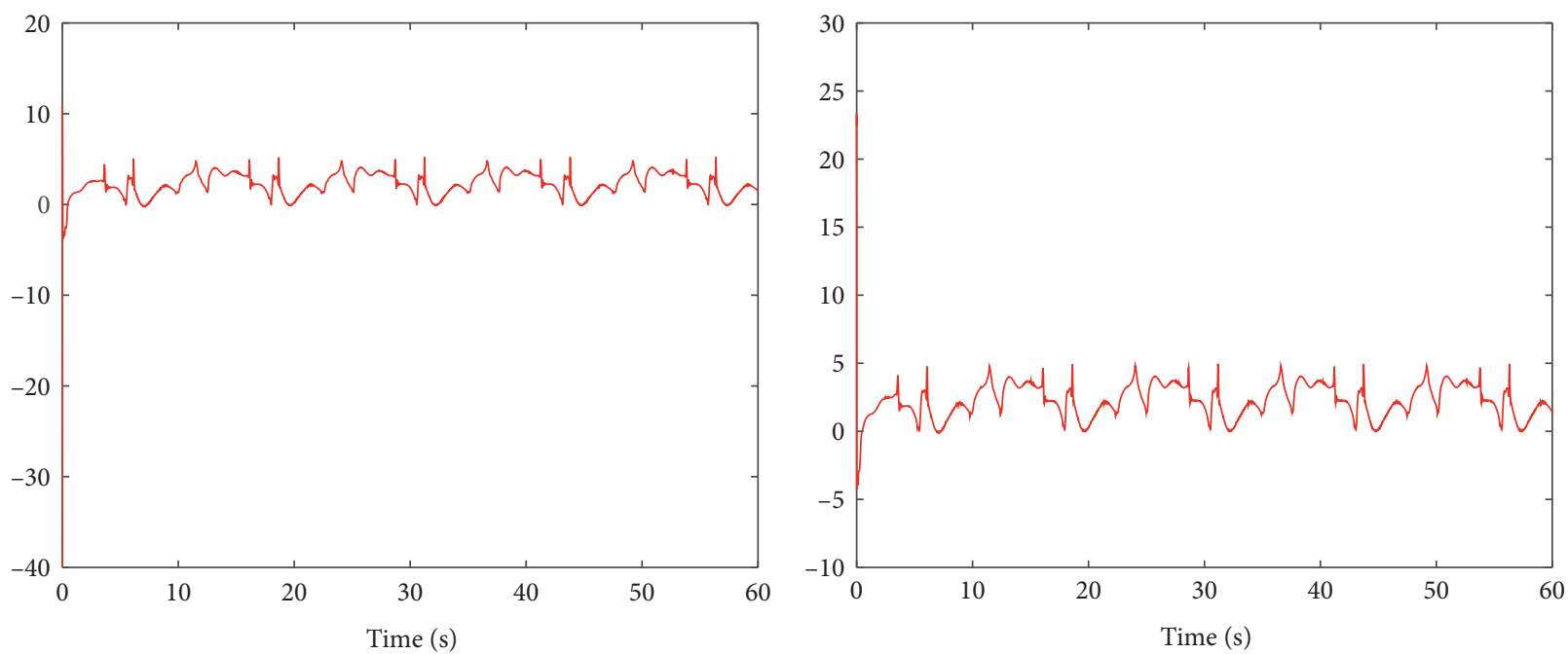

$-u_{1}$

$-u_{2}$

(a)

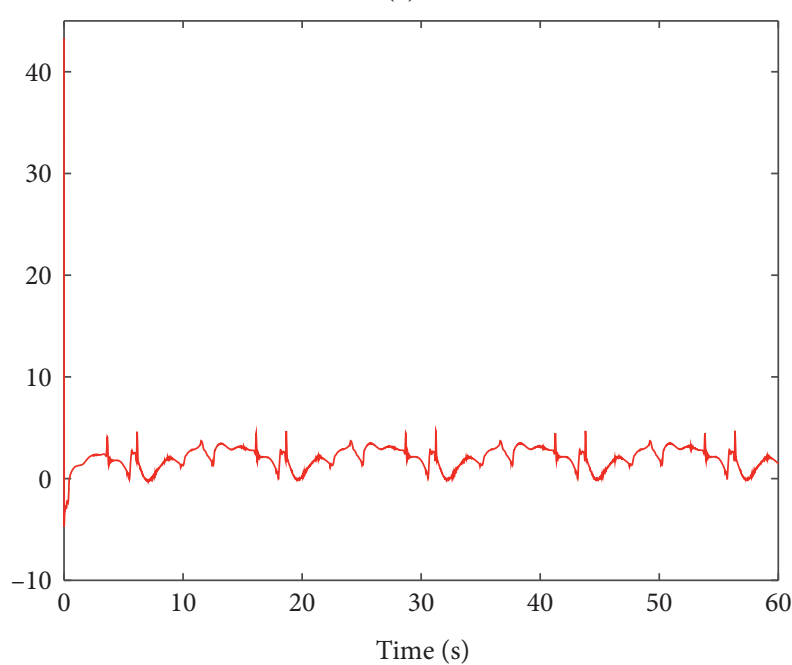

(b)

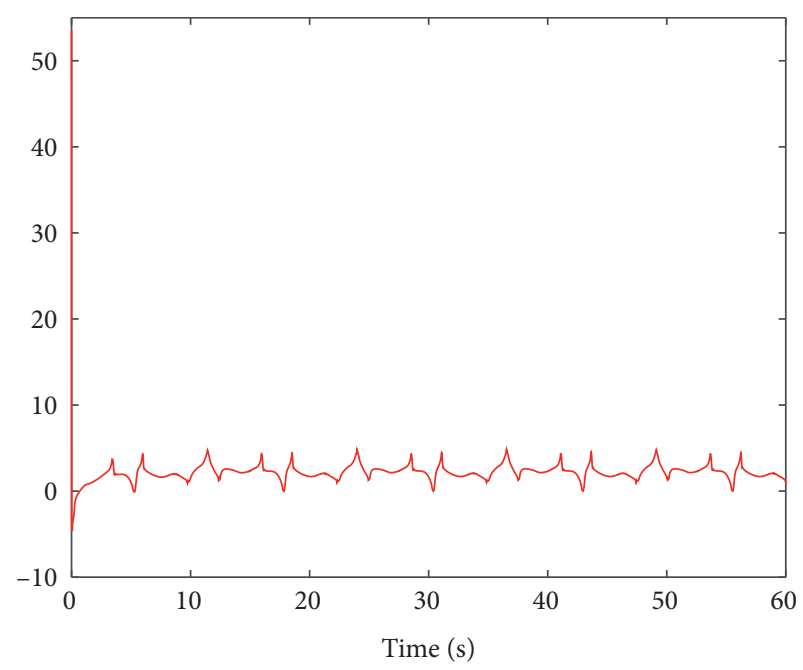

$-u_{3}$

$-u_{4}$

(c)

(d)

FIgure 5: Control inputs in MASs. 


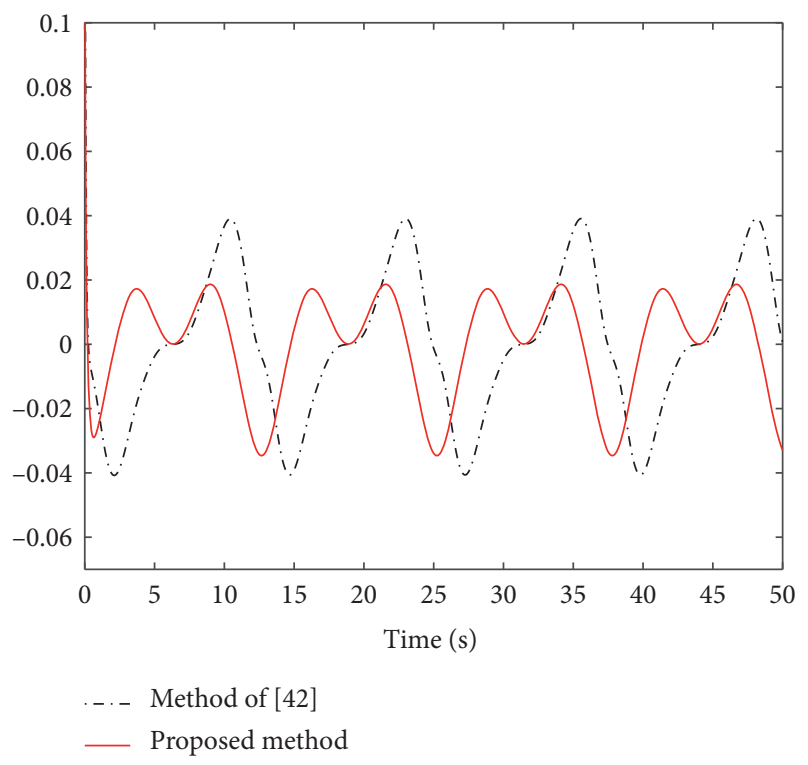

FIGURE 6: Response curves of the tracking errors between this paper and [44].

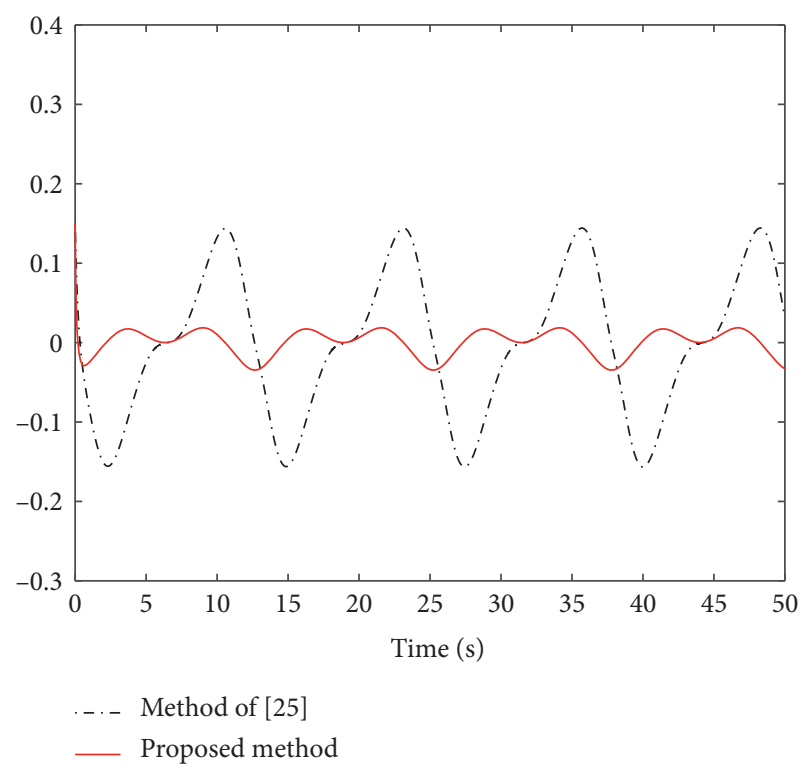

FIGURE 7: Response curves of the tracking errors between this paper and [25].

this example is to design a fixed-time controller to make each follower's output $y_{i}$ track the leader's output signal $y_{0}$ within fixed time.

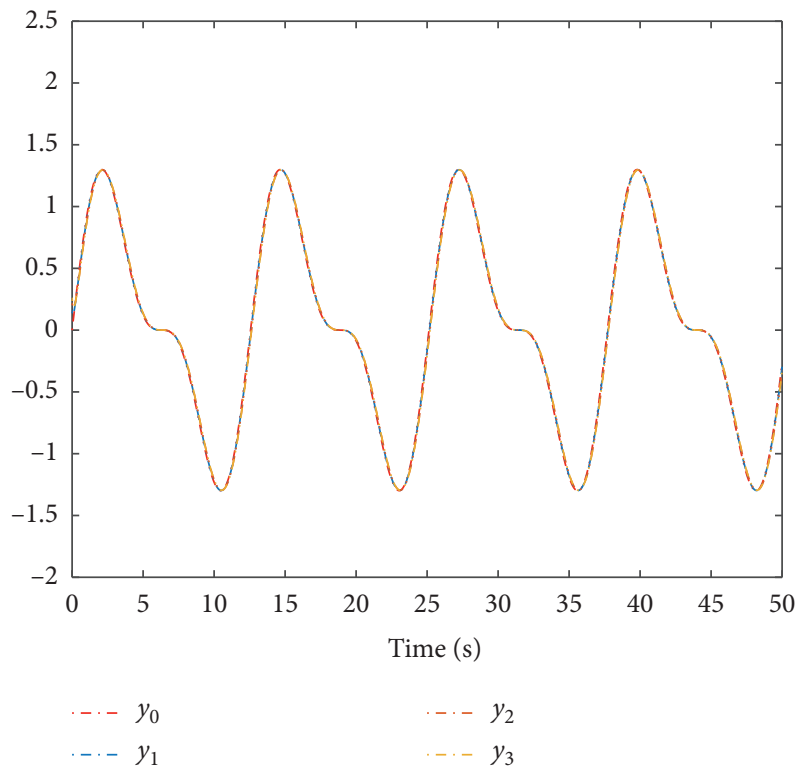

FIgURE 8: Response curves of the output signals $y_{i}$ and $y_{0}$.

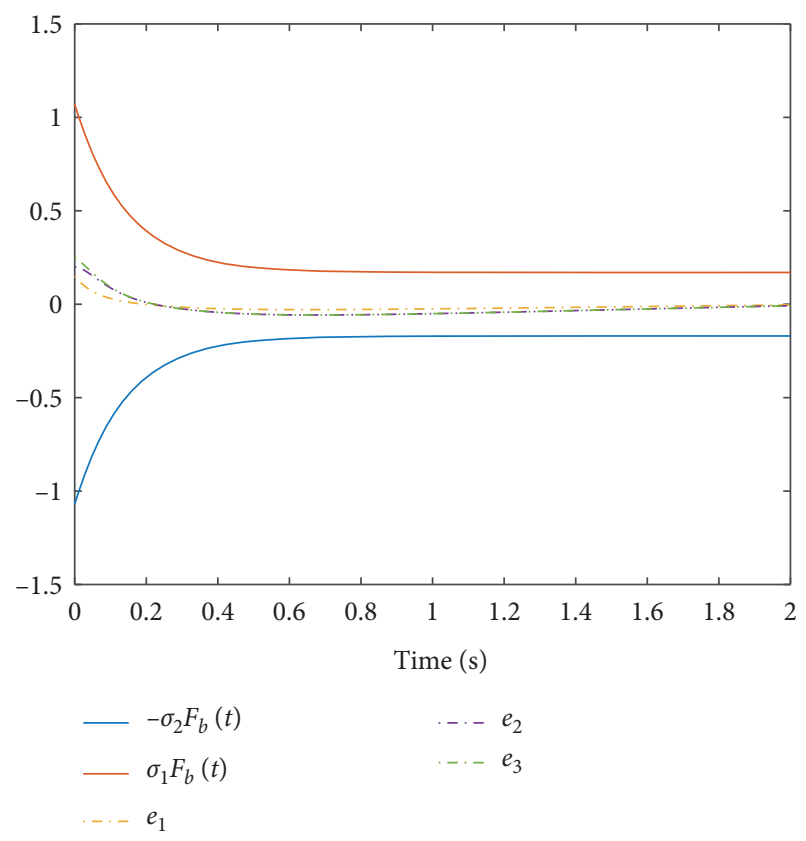

FIGURE 9: Response curves of the tracking consensus error $e_{i}$.

Then, the virtual/actual control signals and the adaptive laws are, respectively, designed as follows: 

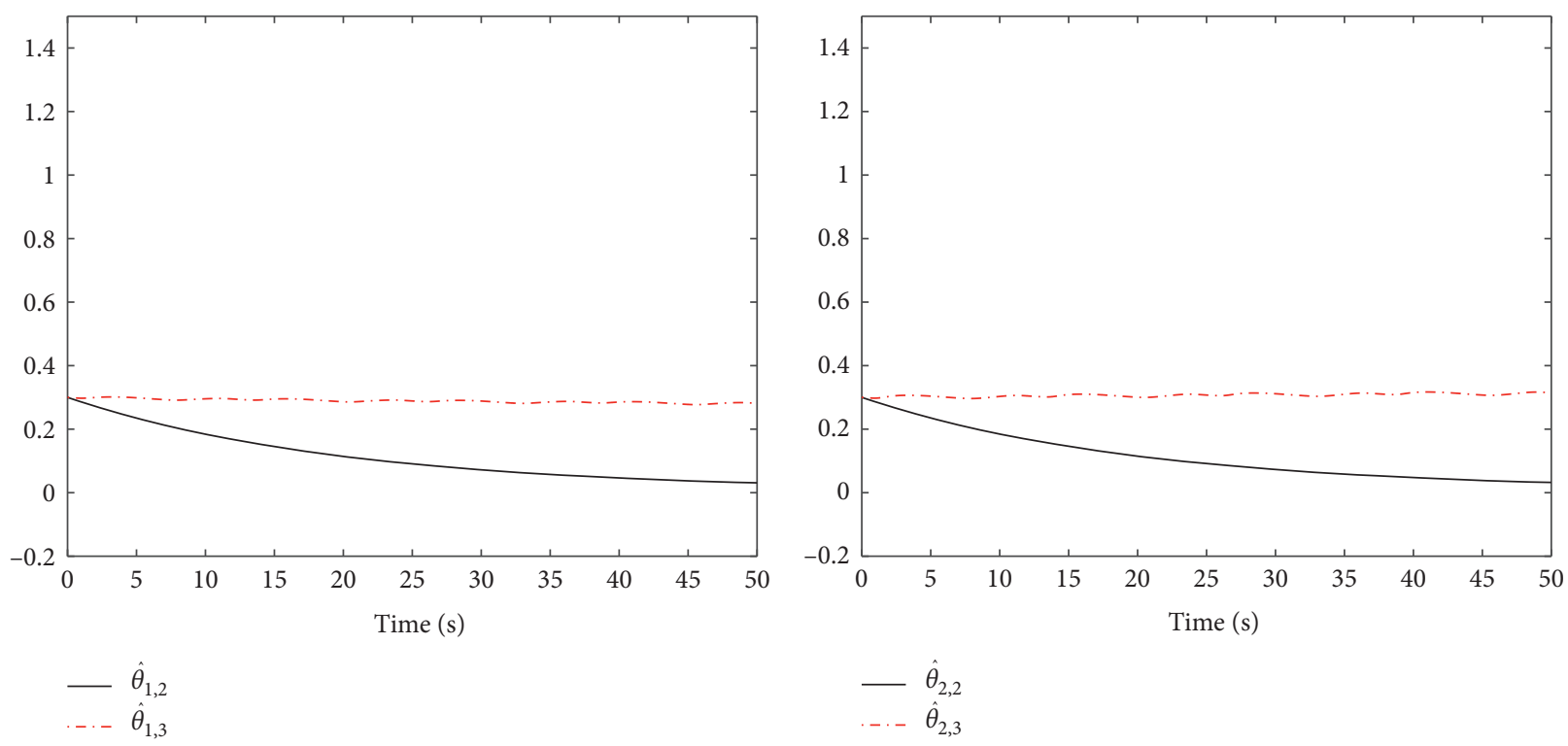

(a)

(b)

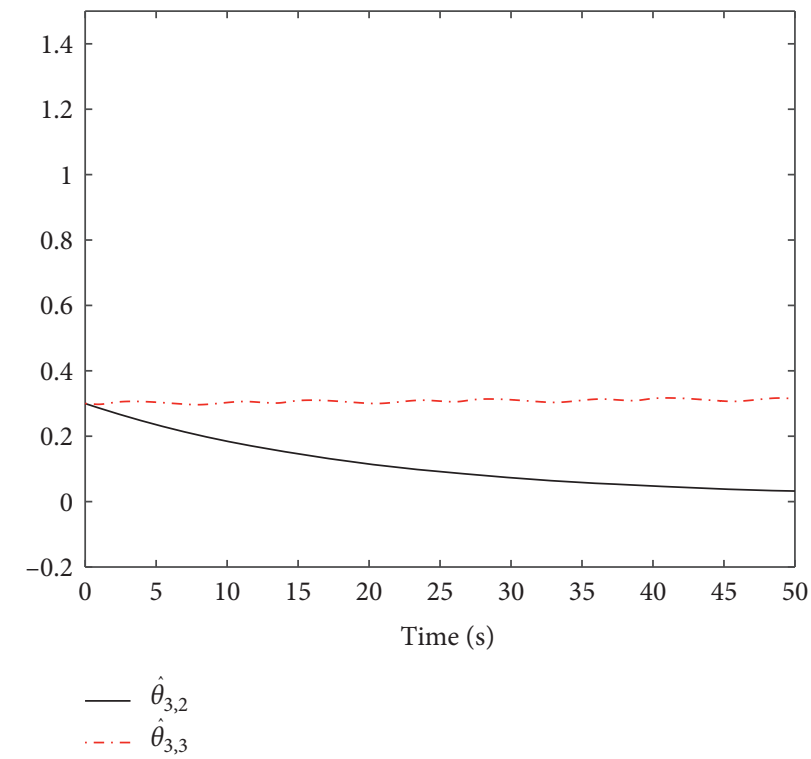

(c)

Figure 10: Adaptive laws in MASs.

$$
\begin{aligned}
v_{i, 1} & =\frac{1}{\left(d_{i}+h_{i}\right) F_{b} \Phi_{F_{i}}}\left(-\zeta_{i, 11} S_{z_{i, 1}, 1}-\zeta_{i, 12} S_{z_{i, 1}, 2}-\zeta_{i, 13} z_{i, 1}\right), \\
v_{i, 2} & =-\frac{\widehat{\theta}_{i, 2}}{2 \eta_{i, 2}^{2}} S_{i, 2}^{T}\left(Z_{i, 2}\right) S_{i, 2}\left(Z_{i, 2}\right) z_{i, 2}+x_{i, 20}-\zeta_{i, 21} S_{z_{i, 2}, 1}-\zeta_{i, 22} S_{z_{i, 2}, 2}-\zeta_{i, 23} z_{i, 2}, \\
u_{i} & =-\zeta_{i, 31} S_{z_{i, 3}, 1}-\zeta_{i, 33} S_{z_{i, 3}, 3}-\frac{\widehat{\theta}_{i, 3}}{3 \eta_{i, 3}^{3}} S_{i, 3}^{T}\left(Z_{i, 3}\right) S_{i, 3}\left(Z_{i, 3}\right) z_{i, 3}-\zeta_{i, 33} z_{i, 3}+x_{i, 30}, \\
\dot{\hat{\theta}}_{i, 2} & =\frac{\gamma_{i}}{2 \eta_{i, 2}^{2}} S_{i, 2}^{T}\left(Z_{i, 2}\right) S_{i, 2}\left(Z_{i, 2}\right) z_{i, 2}^{2}-\lambda_{i} \widehat{\theta}_{i, 2}, \\
\dot{\hat{\theta}}_{i, 3}= & \frac{\gamma_{i}}{2 \eta_{i, 3}^{2}} S_{i, 3}^{T}\left(Z_{i, 3}\right) S_{i, 3}\left(Z_{i, 3}\right) z_{i, 3}^{2}-\lambda_{i} \widehat{\theta}_{i, 3},
\end{aligned}
$$




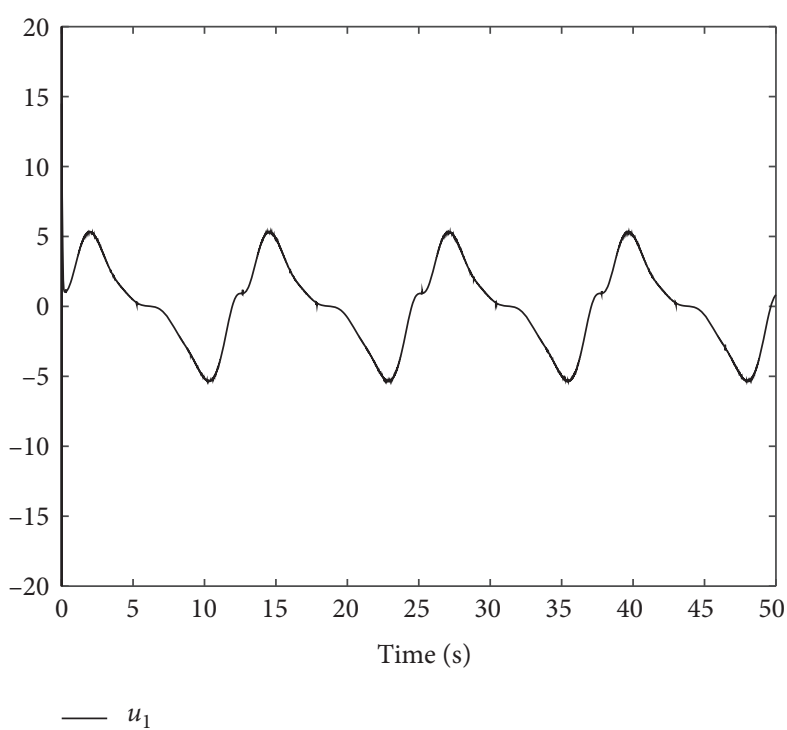

(a)

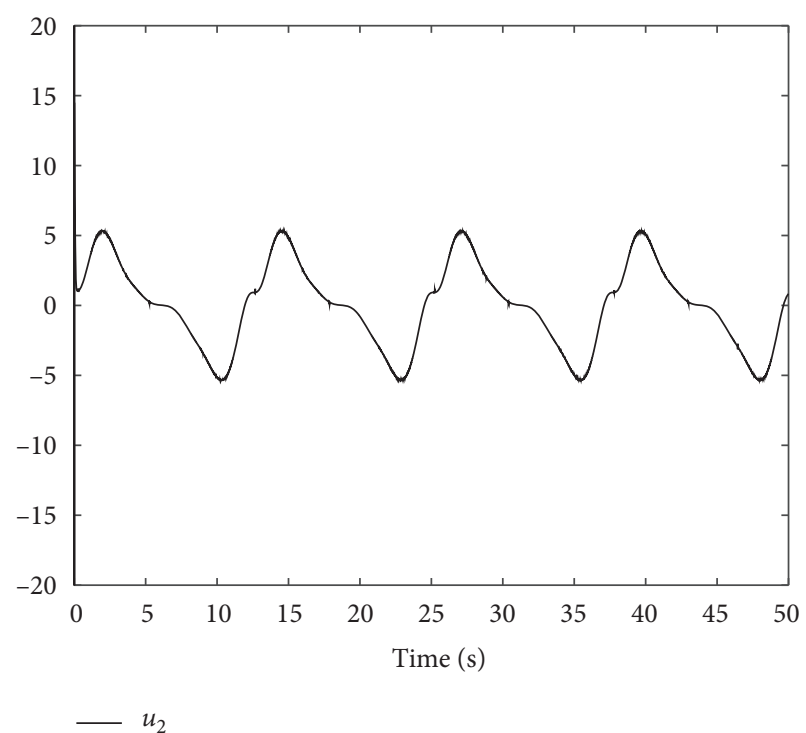

(b)

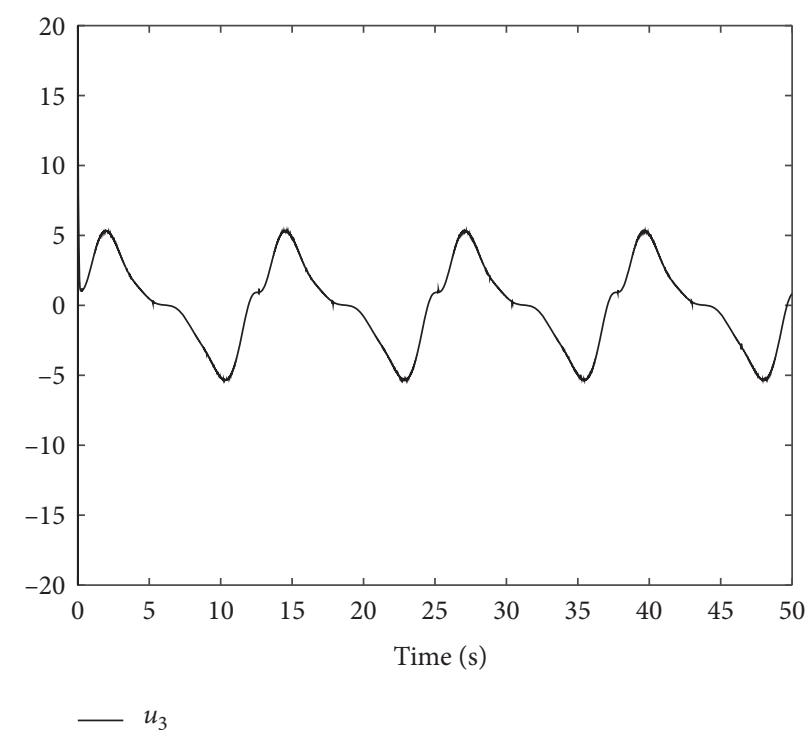

(c)

FIgURe 11: Control inputs in MASs.

where $i=1,2,3$.

To verify the effectiveness of our designed fixed-time consensus control protocol, we compared the tracking performances in system (94) of two existing control strategies that are fixed-time controller in [25] and traditional finite-time controller in [44]. For fair comparison, we choose the same design parameters as follows: $\zeta_{i, 11}=\zeta_{i, 12}=10$, $\zeta_{i, 21}=\zeta_{i, 22}=10, \quad \zeta_{i, 31}=\zeta_{i, 32}=10, \quad \zeta_{i, 23}=\zeta_{i, 33}=60$, $x_{i, 10}=x_{i, 20}=x_{i, 30}=0.1$, and $b_{i}=10$. The initial conditions are $x_{1}(0)=[0.15,0.15,0.15]^{T},\left[x_{2}(0)=[0.2,0.2,0.2]^{T}\right.$, and $\left[x_{3}(0)=[0.25,0.25,0.25]^{T}\right.$, where $x_{i}(0)=\left[x_{i, 1}(0), x_{i, 2}(0)\right.$, $\left.x_{i, 3}(0)\right]$, and $\left[\hat{\theta}_{i, 2}(0), \widehat{\theta}_{i, 3}(0)\right]=[0.3,0.3]^{T}$ with $i=1,2,3$. The design of RBF neural network is the same as in [44].
Remark 7. Since the control protocols in $[25,44]$ are not designed for MASs, we choose the performance of the agent 1 that tracks directly the leader's output to compare, and the comparative results are shown in Figures 6 and 7.

In Figure 8, the tracking curves of the output signals $y_{i}$ are presented. It can be seen that, by using the proposed controller in this paper, the followers can quickly follow the leader's output. Figure 9 shows that each local error is bounded by prescribed functions. In Figures 10 and 11, the responses of adaptive laws and control inputs in MASs are given, respectively. In Figures 6 and 7, the tracking error curves between this paper and $[25,44]$ are presented. It can be seen that the tracking performance by using our 
controller is better than theirs, since the proposed scheme has higher tracking performance with higher accuracy, and the comparison results further illustrate the effectiveness and superiority of the proposed method in this paper.

\section{Conclusion}

In this paper, the adaptive fixed-time tracking consensus problem has been investigated for multiple uncertain nonlinear pure-feedback systems with performance constraint already. Mean value theorem is employed to address the problem of nonaffine structure in pure-feedback nonlinear systems, and RBF neural networks are introduced to approximate unknown nonlinear functions. Based on Lyapunov stability theory, it is proved that the proposed control scheme can ensure all the signals in MASs are bounded, and each local tracking error can converge to a small neighborhood of zero within fixed time. Furthermore, the performance of tracking error is guaranteed to be bounded by the prescribed boundary function. The effectiveness of the work has been proved by stability analysis along with simulation results.

\section{Data Availability}

No data were used to support this study.

\section{Conflicts of Interest}

The authors declare that they have no conflicts of interest.

\section{Authors' Contributions}

Pinwei Li put forward research topics; designed the research plan; drafted the manuscript; and revised the manuscript. Jiyang Dai was involved in guidance support; obtaining research funding; and final review of the manuscript. Jin Ying checked the English grammar and guided the simulation programming.

\section{Acknowledgments}

This study was supported by the National Natural Science Foundation of China under Grant nos. 61663032 and 61663030.

\section{References}

[1] S. P. Bhat and D. S. Bernstein, "Finite-time stability of continuous autonomous systems," SIAM Journal on Control and Optimization, vol. 38, no. 3, pp. 751-766, 2000.

[2] B. Tian, L. Liu, H. Lu, Z. Zuo, Q. Zong, and Y. Zhang, "Multivariable finite time attitude control for quadrotor UAV: theory and experimentation," IEEE Transactions on Industrial Electronics, vol. 65, no. 3, pp. 2567-2577, 2017.

[3] S. Li and X. Wang, "Finite-time consensus and collision avoidance control algorithms for multiple AUVs," Automatica, vol. 49, no. 11, pp. 3359-3367, 2013.

[4] X. Jin, "Fault tolerant finite-time leader-follower formation control for autonomous surface vessels with LOS range and angle constraints," Automatica, vol. 68, pp. 228-236, 2016.
[5] M. Ou, H. Du, and S. Li, "Finite-time tracking control of multiple nonholonomic mobile robots," Journal of the Franklin Institute, vol. 349, no. 9, pp. 2834-2860, 2012.

[6] X. Lu, Y. Wang, X. Yu, and J. Lai, "Finite-time control for robust tracking consensus in MASs with an uncertain leader," IEEE Transactions on Cybernetics, vol. 47, no. 5, pp. 1210$1223,2016$.

[7] X. Wang, S. Li, and P. Shi, "Distributed finite-time containment control for double-integrator multiagent systems," IEEE Transactions on Cybernetics, vol. 44, no. 9, pp. 15181528, 2014.

[8] S. Yu and X. Long, "Finite-time consensus for second-order multi-agent systems with disturbances by integral sliding mode," Automatica, vol. 54, pp. 158-165, 2015.

[9] S. Sui, S. Tong, and C. L. P. Chen, "Finite-time filter decentralized control for nonstrict-feedback nonlinear largescale systems," IEEE Transactions on Fuzzy Systems, vol. 26, no. 6, pp. 3289-3300, 2018.

[10] S. Sui, C. L. P. Chen, and S. Tong, "Neural network filtering control design for nontriangular structure switched nonlinear systems in finite time," IEEE Transactions on Neural Networks and Learning Systems, vol. 30, no. 7, pp. 2153-2162, 2018.

[11] S. Sui, C. L. P. Chen, S. Tong, and S. Feng, "Finite-time adaptive quantized control of stochastic nonlinear systems with input quantization: a broad learning system based identification method," IEEE Transactions on Industrial Electronics, vol. 67, no. 10, pp. 8555-8565, 2019.

[12] L. Wang and F. Xiao, "Finite-time consensus problems for networks of dynamic agents," IEEE Transactions on Automatic Control, vol. 55, no. 4, pp. 950-955, 2010.

[13] H. Du, S. Li, and C. Qian, "Finite-time attitude tracking control of spacecraft with application to attitude synchronization," IEEE Transactions on Automatic Control, vol. 56, no. 11, pp. 2711-2717, 2011.

[14] S. P. Bhat and D. S. Bernstein, "Geometric homogeneity with applications to finite-time stability," Mathematics of Control, Signals, and Systems, vol. 17, no. 2, pp. 101-127, 2005.

[15] S. Li, H. Du, and X. Lin, "Finite-time consensus algorithm for multi-agent systems with double-integrator dynamics," Automatica, vol. 47, no. 8, pp. 1706-1712, 2011.

[16] S. Khoo, L. Xie, S. Zhao, and Z. Man, "Multi-surface sliding control for fast finite-time leader-follower consensus with high order SISO uncertain nonlinear agents," International Journal of Robust and Nonlinear Control, vol. 24, no. 16, pp. 2388-2404, 2014.

[17] Y. Wang, Y. Song, M. Krstic, and C. Wen, "Adaptive finite time coordinated consensus for high-order multi-agent systems: adjustable fraction power feedback approach," Information Sciences, vol. 372, pp. 392-406, 2016.

[18] Y. Zhou, X. Yu, C. Sun, and W. Yu, "Higher order finite-time consensus protocol for heterogeneous multi-agent systems," International Journal of Control, vol. 88, no. 2, pp. 285-294, 2015.

[19] A. Polyakov, "Nonlinear feedback design for fixed-time stabilization of linear control systems," IEEE Transactions on Automatic Control, vol. 57, no. 8, pp. 2106-2110, 2012.

[20] Z. Zuo, Q.-L. Han, B. Ning, X. Ge, and X.-M. Zhang, “An overview of recent advances in fixed-time cooperative control of multiagent systems," IEEE Transactions on Industrial Informatics, vol. 14, no. 6, pp. 2322-2334, 2018.

[21] J. Fu and J. Wang, "Fixed-time coordinated tracking for second-order multi-agent systems with bounded input uncertainties," Systems \& Control Letters, vol. 93, pp. 1-12, 2016. 
[22] J. Ni, L. Liu, C. Liu, and J. Liu, "Fixed-time leader-following consensus for second-order multiagent systems with input delay," IEEE Transactions on Industrial Electronics, vol. 64, no. 11, pp. 8635-8646, 2017.

[23] H. Li, M. Zhu, Z. Chu, H. Du, G. Wen, and N. D. Alotaibi, "Fixed-time synchronization of a class of second-order nonlinear leader-following multi-agent systems," Asian Journal of Control, vol. 20, no. 1, pp. 39-48, 2018.

[24] Z. Y. Zuo, "Leader-follower fixed-time consensus of multiagent systems with high-order integrator dynamics," International Journal of Control, vol. 90, no. 7, pp. 1420-1427, 2017.

[25] Z. Zuo, B. Tian, M. Defoort, and Z. Ding, "Fixed-time consensus tracking for multiagent systems with high-order integrator dynamics," IEEE Transactions on Automatic Control, vol. 63, no. 2, pp. 563-570, 2018.

[26] H. Yang and D. Ye, "Adaptive fixed-time bipartite tracking consensus control for unknown nonlinear multi-agent systems: an information classification mechanism," Information Sciences, vol. 459, pp. 238-254, 2018.

[27] H. Yang and D. Ye, "Distributed fixed-time consensus tracking control of uncertain nonlinear multiagent systems: a prioritized strategy," IEEE Transactions on Cybernetics, vol. 50, no. 6, pp. 2627-2638, June 2020.

[28] H. Wang, W. Yu, G. Wen, and G. Chen, "Fixed-time consensus of nonlinear multi-agent systems with general directed topologies," IEEE Transactions on Circuits and Systems II: Express Briefs, vol. 66, no. 9, pp. 1587-1591, 2019.

[29] Y. Wu, Z. Wang, and Z. Huang, "Distributed fault detection for nonlinear multi-agent systems under fixed-time observer," Journal of the Franklin Institute, vol. 356, no. 13, pp. 75157532, 2019.

[30] L. Zifu, L. Tieshan, F. Gang, Z. Rong, and S. Qihe, "Neural network-based adaptive control for pure-feedback stochastic nonlinear systems with time-varying delays and dead-zone input," IEEE Transactions on Systems, Man, and Cybernetics: Systems, vol. 50, no. 12, 2020.

[31] W. Liu, Q. Ma, G. Zhuang, J. Lu, and Y. Chu, “An improved adaptive neural dynamic surface control for pure-feedback systems with full state constraints and disturbance," Applied Mathematics And Computation, vol. 358, pp. 37-50, Oct. 2019.

[32] Y. Sun, B. Chen, F. Wang, S. Zhou, and H. Wang, "A novel adaptive control method for a class of stochastic switched pure feedback systems," Neurocomputing, vol. 367, pp. 337345, Nov. 2019.

[33] G. Wang, C. Wang, and L. Li, "Fully distributed low-complexity control for nonlinear strict-feedback multiagent systems with unknown dead-zone inputs," IEEE Transactions on Systems, Man, and Cybernetics: Systems, vol. 50, no. 2, pp. 421-431, 2017.

[34] G. Wang, C. Wang, and X. Cai, "Consensus control of outputconstrained multiagent systems with unknown control directions under a directed graph," International Journal of Robust and Nonlinear Control, vol. 30, no. 5, pp. 1802-1818, 2020.

[35] G. Wang, Y. Ji, and C. Wang, “Asymptotic tracking control with preassigned transient performance for strict-feedback systems in the presence of unknown control directions," Journal of the Franklin Institute, vol. 357, no. 1, pp. 206-228, 2020.

[36] C. Hua, G. Liu, L. Zhang, and X. Guan, "Output feedback tracking control for nonlinear time-delay systems with tracking errors and input constraints," Neurocomputing, vol. 173, pp. 751-758, 2016.
[37] M. Wang, S. S. Ge, and K.-S. Hong, “Approximation-based adaptive tracking control of pure-feedback nonlinear systems with multiple unknown time-varying delays," IEEE Transactions on Neural Networks, vol. 21, no. 11, pp. 1804-1816, Nov. 2010.

[38] R. M. Sanner and J.-J. E. Slotine, "Gaussian networks for direct adaptive control," IEEE Transactions on Neural Networks, vol. 3, no. 6, pp. 837-863, 1992.

[39] D. Ba, Y.-X. Li, and S. Tong, "Fixed-time adaptive neural tracking control for a class of uncertain nonstrict nonlinear systems," Neurocomputing, vol. 363, pp. 273-280, 2019.

[40] A. J. Kurdila, F. J. Narcowich, and J. D. Ward, "Persistency of excitation in identification using radial basis function approximants," SIAM Journal on Control and Optimization, vol. 33, no. 2, pp. 625-642, 1995.

[41] A. F. Filippov, Differential Equations with Discontinuous Right hand Sides: Control Systems, Vol. 33, Springer Science \& Business Media, Berlin, Germany, 2013.

[42] Z. Zuo, "Nonsingular fixed-time consensus tracking for second-order multi-agent networks," Automatica, vol. 54, pp. 305-309, 2015.

[43] C. Wang and Y. Lin, "Decentralized adaptive tracking control for a class of interconnected nonlinear time-varying systems," Automatica, vol. 54, pp. 16-24, Apr. 2015.

[44] Y. Sun, B. Chen, C. Lin, and H. Wang, "Finite-time adaptive control for a class of nonlinear systems with nonstrict feedback structure," IEEE Transactions on Cybernetics, vol. 48, no. 10, pp. 2774-2782, 2018. 